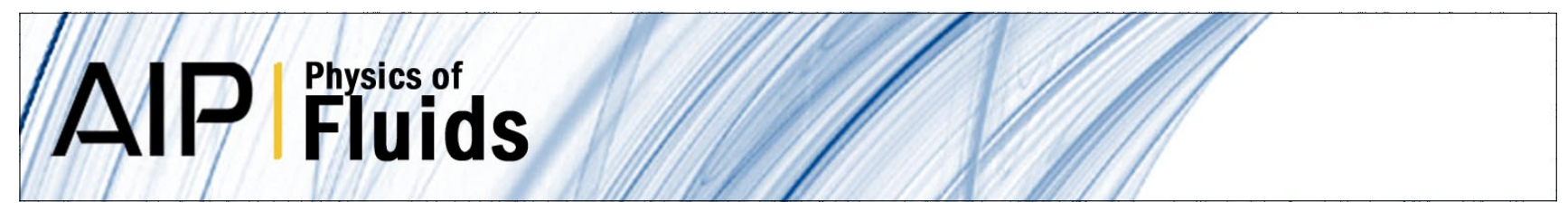

\title{
On the stick-slip flow from slit and cylindrical dies of a Phan-Thien and Tanner fluid model. II. Linear stability analysis
}

George Karapetsas and John Tsamopoulos

Citation: Phys. Fluids 25, 093105 (2013); doi: 10.1063/1.4821805

View online: http://dx.doi.org/10.1063/1.4821805

View Table of Contents: http://pof.aip.org/resource/1/PHFLE6/v25/i9

Published by the AIP Publishing LLC.

\section{Additional information on Phys. Fluids}

Journal Homepage: http://pof.aip.org/

Journal Information: http://pof.aip.org/about/about_the_journal

Top downloads: http://pof.aip.org/features/most_downloaded

Information for Authors: http://pof.aip.org/authors

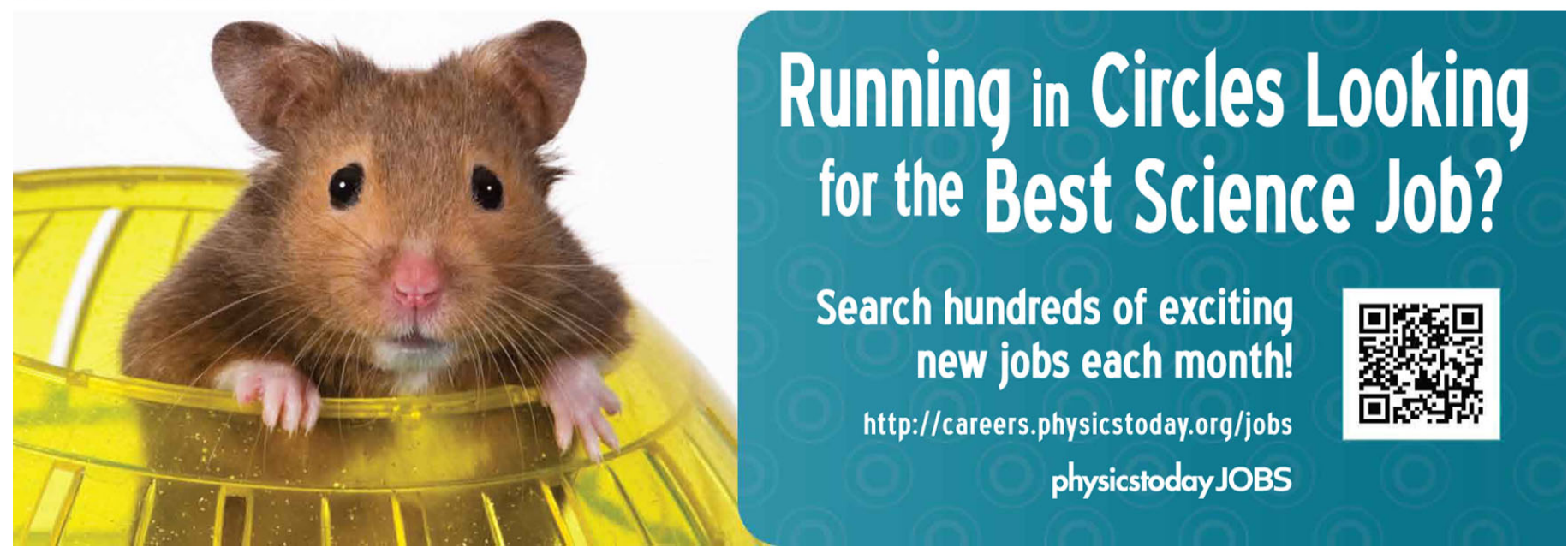




\title{
On the stick-slip flow from slit and cylindrical dies of a Phan-Thien and Tanner fluid model. II. Linear stability analysis
}

\author{
George Karapetsas ${ }^{1, a)}$ and John Tsamopoulos ${ }^{2, b)}$ \\ ${ }^{1}$ Department of Mechanical Engineering, University of Thessaly, Volos 38334, Greece \\ ${ }^{2}$ Department of Chemical Engineering, University of Patras, Patras 26500, Greece
}

(Received 24 April 2013; accepted 30 August 2013; published online 27 September 2013)

\begin{abstract}
During extrusion of viscoelastic fluids various flow instabilities may arise resulting in a distorted free surface. In order to investigate the factors generating these instabilities we performed a linear stability analysis at zero Reynolds number around the steady solution of the cylindrical or planar stick-slip flow for a viscoelastic fluid following the affine exponential Phan-Thien Tanner (PTT) model. Stick-slip flow is an important special case of the extrudate swell problem, since the latter reduces to it in the limit of infinite surface tension but avoids the complications of a free-boundary flow. The linear stability analysis is performed for various values of the rheological parameters of the PTT model in order to determine the effects of all material properties. It is found that the flow becomes unstable as the Weissenberg number increases above a critical value, due to a Hopf bifurcation suggesting that the flow will become periodic in time. Both the critical value of the Weissenberg number and the frequency of the instability depend strongly on the rheological parameters of the viscoelastic model. The corresponding eigenvectors indicate that the perturbed flow field has a spatially periodic structure, initiated at the rim of the die, extending for up to 5-7 die gaps downstream, but confined close to the surface of the extrudate, in qualitative agreement with existing experiments. This suggests that instability is generated by the combination of the singularity in the velocity and stress fields at the die lip and the strong extension that the extruded polymer undergoes near its surface. The elasticity alone can be responsible for the appearance of instabilities in the extrusion process of viscoelastic fluids and the often used assumptions of wall slip or compressibility, although they might be present, are not required. Finally, the mechanisms that produce these instabilities are examined through energy analysis of the disturbance flow.
\end{abstract} (c) 2013 AIP Publishing LLC. [http://dx.doi.org/10.1063/1.4821805]

\section{INTRODUCTION}

The extrusion process is widely used in the polymer industry. It is well known that during this process a viscoelastic fluid experiences significant swelling while various flow instabilities may arise. From industrial practice and laboratory experiments, it is known that below a critical shear stress the surface of the extrudate is smooth, whereas beyond this critical shear stress (around 0.1-0.3 MPa for many polymers) the surface becomes distorted ${ }^{1-7}$ First the extrudate surface exhibits a smallamplitude, high-frequency disturbance in the form of local cracks, which form longitudinal bands that increase in width and number. This is generally known as sharkskin. At higher shear stresses it exhibits alternating smooth and distorted sections; this is known as stick-slip or spurt flow. Upon further increase of the shear stress, gross irregularities develop even in the bulk of the extrudate, often called melt fracture. We should note, however, that the nature of the surface distortions that arise

\footnotetext{
a) Email: gkarapetsas@gmail.com

b)Email: tsamo@chemeng.upatras.gr
} 
strongly depends on the polymer, the presence of a solvent and the die material. ${ }^{7}$ These deformations have been attributed to instabilities, the origin of which is still unclear.

The onset of such flow instabilities, as expected, affects significantly the quality of the final product and therefore imposes a limit on the production rate in many polymer processing operations. It is not surprising therefore that several researchers have dealt with this problem in the past performing very careful and revealing experiments in their attempt to enhance our understanding on this difficult problem. ${ }^{8-15}$ Over the last 30 years several theories for the origin of these instabilities have been put forth. Among them three have prevailed till now. The first one associates the visual appearance of the surface defect with the loss of adhesion at the polymer-wall interface inside the die, the second one attributes it to the strong stress field that develops right at the die exit and the intense extensional flow especially at the surface of the material that follows, while according to the third one the extrudate distortions could arise from an inherent instability of the constitutive model. ${ }^{1-6}$ The association of the sharkskin appearance with the onset of slip was indicated by the early experiments of Ramamurthy ${ }^{8}$ together with the observation that the visual onset of the sharkskin instability corresponds to a change in the slope of the flow curve and studies of the effect of the ratio of the die length $\mathrm{L}$ over its diameter $\mathrm{D}$ on the flow curve. ${ }^{9}$ This scenario has been revisited by many research groups since then, but it is contradicted by the fact that the onset of such instabilities could be delayed significantly by using materials in the polymer or the die wall that promote slip of the fluid with respect to the die with very good results in the quality of the product. Moreover, slip is not always present in the sharkskin regime, since the experiments of El Kissi et al. ${ }^{11,12}$ showed wall slip to be negligible, whereas sharkskin effects were observed to initiate at the die exit and diminish with the distance from it. The same authors attributed the change of slope in the flow curve to shear thinning of the fluid and found no effect of the L/D ratio on it.

As far as the stick-slip instability is concerned, the theoretical explanations suggested in the literature are based on the non-monotonicity of the flow curve and the fact that steady state solutions corresponding to the negative slope regime of the flow curve are unstable. It has been suggested that such a flow curve can be obtained either by a non-monotonic slip-law or by a non-monotonic constitutive equation. ${ }^{2-4,6}$ These theoretical studies are still under thorough examination, in spite the sometimes controversial assumptions involved. Although the above mechanisms are very different in principle, it is very difficult to distinguish them in practice. This is exactly the aspect where the theoretical approach could play an important role in order to improve our understanding of this problem. This is also the purpose of the present work.

A first attempt to examine possible mechanisms for the stick-slip instability was made by Georgiou and Crochet. ${ }^{16}$ To this end, they solved the time-dependent compressible Newtonian extrudate swell problem considering an arbitrary non-monotonic slip model at the die wall. Their dynamic simulations indeed showed that the flow inside the die was oscillatory causing the shape of the free surface to become wavy. One question that arises is whether compressibility is a necessary condition for the instability to appear. This was examined by Brasseur et al. ${ }^{17}$ who presented time dependent simulations for an incompressible Oldroyd-B fluid with the same non-monotonic slip model. It was shown that indeed viscoelasticity may replace compressibility, and when combined with a non-monotonic slip law it can act as storage of elastic energy generating self-sustained pressure oscillations and waves on the extrudate surface in the stick-slip regime. However, it has been found that these two mechanisms can lead to different type of oscillations. The first one leads to oscillations similar to the stick-slip instability whereas the second one leads to small amplitude high-frequency oscillations that look like sharkskin. ${ }^{6}$ Moreover it has been suggested that the latter oscillations may be superimposed to the ones caused by the compressibility/slip mechanism.

The second possible mechanism for the sharkskin instability claims that it results from the coupling of the strong and primarily extensional stress at the die exit which leads to cracking of the fluid there. It was first suggested by Cogswell ${ }^{15}$ and partially confirmed by experiments by Kissi et al. ${ }^{12}$ Migler et al.,${ }^{18}$ among others. The experiments in Ref. 18 in particular demonstrate that there is no disturbance of the velocity within $20 \mu \mathrm{m}$ from the die exit, thus refuting the idea that stick-slip transitions inside the die are necessary to initiate the instability. Moreover, they present high-speed photographs of the sharkskin cycle for PE, which show the splitting of the polymer into two layers near the exit lip. In fact it has been proposed that the definition of sharkskin should be given to an 
instability initiated at the die exit. ${ }^{6}$ Our analysis and results reported subsequently support, at least qualitatively, this mechanism in several ways, so we will not expand on it here.

The third mechanism which is under investigation is whether these process instabilities may arise due to inherent constitutive instabilities. As it was noted earlier it has been argued that in order for the flow to become unstable the constitutive law should be non-monotonic, i.e., exhibit non-monotonicity of the shear stress/shear rate curve in simple shear Poiseuille flows. ${ }^{2}$ Although this mechanism was shown to lead to an unstable flow, ${ }^{19}$ it has also received a lot of criticism by several researchers as inappropriate to describe the stick-slip instability. ${ }^{6,20,21}$ Nevertheless, it is a fact that purely elastic instabilities have been reported in the past in viscometric flows of viscoelastic fluids. A nice review on this subject has been presented by Shaqfeh. ${ }^{22}$

Black and Graham ${ }^{23}$ proposed another mechanism that can lead to short-wave flow instabilities in plane shear flows. These authors have considered the PTT viscoelastic model together with a slip model which includes not only shear stress but also normal stress dependence of the slip velocity. Although both the constitutive as well as the slip model used are monotonic it was shown that instability may arise due to the interaction of slip and viscoelasticity. Clearly, the findings of this work show that the non-monotonicity is not an essential condition for the appearance of instabilities.

Taking all these into consideration we have decided to examine whether widely acceptable constitutive laws (i.e., with monotonic flow curves) without the presence of any slip can predict the sharkskin instability, which is the first problem to solve since it appears first as the Weissenberg number increases and if so which fluid properties promote it. To this end, we have decided to solve the steady viscoelastic stick-slip flow (see Paper I of this study ${ }^{24}$ ) and then to perform a linear stability analysis around this base state to find out whether this solution is stable or not. This is a classical method that has been applied with success in the past to examine the stability in numerous problems of both Newtonian and non-Newtonian flows. ${ }^{25-30}$ We have chosen to work with a monotonic constitutive model in order to find out whether the non-monotonicity is essential for the flow to become unstable. Such a model is the affine Phan-Thien and Tanner model, ${ }^{31,32}$ which is based on network theory. With only one additional parameter to fluid elasticity and viscosity, it can predict a finite extensional viscosity which is either slightly varying with increasing the extension rate (in the linear version of the model) or extensional hardening followed by extensional thinning (in its exponential version) and shear thinning. All these effects are observed in the majority of viscoelastic fluids. Moreover we have chosen to work with the stick-slip flow as it is an important special case since it is formally equivalent to the free surface problem in the limit of infinite surface tension where the normal stress balance reduces to the equation of zero curvature in the flow direction. Therefore this flow has many of the ingredients of the more important extrudate swell problem, i.e., the fact that the boundary conditions change abruptly from no-slip along the wall to perfect slip along the free surface, but without the complication of an unknown free surface.

The main objective of this paper is to examine the stability of the steady viscoelastic twodimensional and axisymmetric stick-slip flow, which has been presented in Paper I of this study. ${ }^{24}$ The rest of this paper is organized as follows. We briefly present the essential features of the linear stability analysis in Sec. II. In Sec. III, we examine the accuracy of our results by performing a thorough mesh refinement study and then proceed in a parametric study to examine the effect of the various rheological parameters of the PTT model on the stability of the flow. Following this, an energy analysis is performed in order to investigate the mechanism of the instability and stability to three-dimensional disturbances or of the reverse flow is examined. Finally, conclusions are drawn in Sec. IV.

\section{LINEAR STABILITY ANALYSIS}

\section{A. Governing equations}

In Paper I of this study ${ }^{24}$ we have examined the steady stick-slip flow of a PTT fluid, either in a cylindrical or a slit die under creeping flow conditions. The viscoelastic fluid is assumed to be incompressible with constant density $\rho$, relaxation time $\lambda$ and total zero-shear viscosity $\mu=\mu_{s}+\mu_{p}$, where $\mu_{s}$ and $\mu_{p}$ are the solvent and polymer contributions, respectively. All lengths 
are scaled with the half of the die gap or the die radius, $R$, velocities with the mean velocity at the inflow boundary, $V$, while the viscous scale is used for both the pressure, $P$, and stress components, $\tau_{i j}$. The flow is characterized by the Weissenberg number, $W i=\lambda V / R$, the ratio of the Newtonian solvent viscosity over the total viscosity, $\beta=\mu_{s} / \mu$, and the ratios, $l_{1}=L_{1} / R$ and $l_{2}=L_{2} / R$, of the distance of the inflow and outflow boundaries from the die exit, $L_{1}$ and $L_{2}$, respectively. Both distances are chosen to be long enough so that they do not affect the flow between them in any way. The steady state equations are discretized using the finite element method and solved using the EVSS-G formulation. ${ }^{33}$ According to it, having decomposed the total stress $\tau$ into contributions from the solvent $2 \beta \underline{\underline{\gamma}}$ and the polymer, $\underline{\underline{\tau}}$, the latter is split further into a purely elastic contribution, $\underline{\underline{\Sigma}}$, and a viscous part,

$$
\underline{\underline{\tau}}=2 \beta \underline{\underline{\underline{\gamma}}}+\stackrel{\tau}{=} p, \quad \underline{\underline{\tau}} p=\underline{\underline{\Sigma}}+2(1-\beta) \underline{\underline{\dot{\gamma}}}
$$

Moreover, an independent interpolation of the components of the velocity gradient tensor is introduced:

$$
\underline{\underline{G}}=\underline{\nabla v}
$$

The former splitting ensures the elliptic nature of the momentum equations even in the absence of a solvent $(\beta=0)$, while the latter substitution makes the approximations in the constitutive equation of the elastic stress and the velocity gradient compatible to each other. The obtained steady state solutions have been meticulously tested for convergence everywhere in the flow domain and especially near the singular point at the exit of the die.

Our goal here is to study the stability of this flow. In order to achieve that, we consider the stability of the steady flow subjected to infinitesimal two- or three-dimensional perturbations. The flow variables are decomposed into a base state and its perturbation using the following ansatz:

$$
\left[\begin{array}{c}
\underline{v}(r, z, t) \\
P(r, z, t) \\
\underline{\underline{G}}(r, z, t) \\
\underline{\underline{\underline{\Sigma}}}(r, z, t)
\end{array}\right]=\left[\begin{array}{c}
\underline{v}_{b}(r, z) \\
P_{b}(r, z) \\
\underline{\underline{G}}_{b}(r, z) \\
\underline{\underline{\underline{\Sigma}}}_{b}(r, z)
\end{array}\right]+\delta\left[\begin{array}{c}
\underline{\hat{v}}(r, z, \theta, t) \\
\hat{P}(r, z, \theta, t) \\
\hat{\underline{\hat{G}}}(r, z, \theta, t) \\
\underline{\underline{\hat{\Sigma}}}(r, z, \theta, t)
\end{array}\right] .
$$

The coordinates given above indicate, the flow direction, $z$, the velocity gradient direction, $r$, or the vorticity direction, $\theta$, in both die geometries. The first terms on the right hand side of this equation represent the steady state solution, indicated by the subscript $b$, while the second ones are the perturbation, indicated by "^" over the corresponding variable. This perturbation is decomposed into Fourier components in the vorticity direction

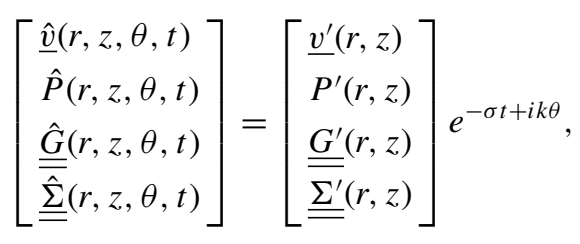

where $k$ is the wavenumber of the perturbation and $\sigma$ is the growth rate. If the calculated $\sigma$ turns out to have a negative real part, the corresponding steady state is unstable. Substituting these expressions into the time-dependent form of the governing equations and neglecting terms of order higher than the first in the perturbation parameter $\delta$, the following set of linearized equations is obtained from the corresponding momentum and mass balances and the PTT model, respectively,

$$
\begin{aligned}
& \underline{\nabla} \hat{P}-\underline{\nabla} \cdot \underline{\underline{\hat{\Sigma}}}-\underline{\nabla} \cdot\left(\underline{\nabla \hat{v}}+\underline{\nabla \hat{v}}^{T}\right)=0, \\
& \underline{\nabla} \cdot \underline{\hat{v}}=0,
\end{aligned}
$$

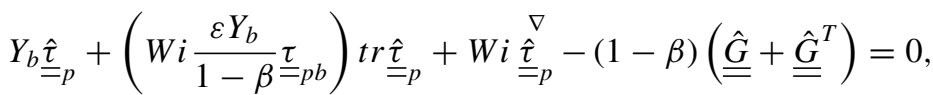


where $Y_{b}=\exp \left[\frac{\varepsilon}{1-\beta} W i \operatorname{tr} \underline{\tau}_{p b}\right]$ stands for the exponential form of the PTT model. ${ }^{32}$ The symbol " $\nabla$ " over the viscoelastic stress denotes the upper convected derivative, defined as

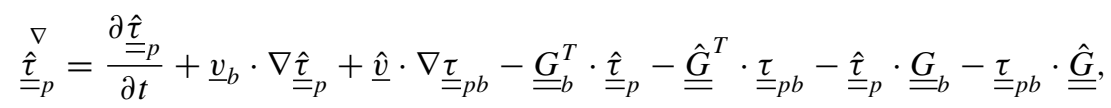

and the expression for the base state and perturbation of the polymeric stress is readily obtained from

$$
\underline{\underline{\tau}}_{p b}=\underline{\underline{\Sigma}}_{b}+(1-\beta)\left(\underline{\underline{G}}_{b}+\underline{\underline{G}}_{b}^{T}\right), \underline{\underline{\hat{\tau}}}_{p}=\underline{\underline{\hat{\hat{v}}}}+(1-\beta)\left(\underline{\underline{\underline{\hat{G}}}}+\underline{\underline{\underline{\hat{G}}}}^{T}\right) .
$$

First, two-dimensional disturbances to the base flow are examined (i.e., for $k=0$ ). Then, the above system of equations is subjected to the following boundary conditions:

$$
\begin{array}{ll}
\hat{v}_{r}=0, \hat{v}_{z}=0, & \text { at } r=1 \text { and } 0 \leq z \leq l_{1}, \\
\hat{v}_{r}=0, \hat{\tau}_{r z}=0, & \text { at } r=1 \text { and } l_{1} \leq z \leq l_{2}, \\
\hat{v}_{r}=0, \hat{v}_{z}=0, \hat{\underline{t}}_{p}=0, & \text { at } z=0, \\
\hat{v}_{r}=0, \frac{\partial \hat{v}_{z}}{\partial z}=0, & \text { at } z=l_{1}+l_{2}, \\
\hat{v}_{r}=0, \frac{\partial \hat{v}_{z}}{\partial r}=0, & \text { at } r=0 .
\end{array}
$$

\section{B. The Arnoldi algorithm}

The above set of equations is discretized using the mixed finite element method, i.e., the finite element representation is constructed from Lagrangian biquadratic approximations for the velocity components and bilinear approximations for the pressure, polymer stress and velocity gradient interpolations. The weak form of the equations is obtained using the SUPG weighting for the hyperbolic constitutive equation and the Galerkin method for the remaining equations. This procedure results in a generalized eigenvalue problem of the form

$$
\underline{\underline{J}} \underline{x}=\sigma \underline{\underline{M}} \underline{x},
$$

where $\underline{\underline{J}}$ and $\underline{\underline{M}}$ are the Jacobian and the mass matrix, respectively, $\sigma$ are the eigenvalues and $\underline{x}$ are the corresponding eigenvectors.

This eigenvalue problem is solved using Arnoldi's method, ${ }^{34}$ which systematically constructs a Krylov sequence to approximate the eigenvectors of a large eigenvalue problem in terms of the eigenvectors of a much smaller problem. Thus it locates only the eigenvalues of interest, i.e., those eigenvalues with the smallest real part. For implementing this algorithm the public domain code ARPACK ${ }^{35}$ is used. This code is capable by default to compute the eigenvalues with the largest magnitude and not those with the smallest real part. Since we are interested only in the latter eigenvalues and to avoid the singularity of the mass matrix, the following shift-and-invert transformation is employed:

$$
(\underline{\underline{J}}-\lambda \underline{\underline{M}})^{-1} \underline{\underline{M}} \underline{x}=v \underline{x}
$$

where $v=\frac{1}{\sigma-\lambda}$.

This transformation is effective for finding eigenvalues near $\lambda$ since the eigenvalues of the transformed problem, $v$, that are largest in magnitude correspond to eigenvalues of the original problem that are nearest to each shift value, $\lambda$. Therefore with a sequence of such complex shifts, 
which is adaptively generated with a procedure similar to the one described in Natarajan ${ }^{36}$ it is possible to obtain the desired part of the eigenspectrum (i.e., the leading eigenvalues with the smaller real part). Typically a sequence of seven different shifts is used, calculating 50 eigenvalues each time. The computations are initiated using the shift $\lambda=0$, since we are interested in the eigenvalues close to the imaginary axis and are continued setting the next shift to $\lambda=\lambda_{I} i$, where $\lambda_{I}$ is a value close to the largest imaginary part of the computed eigenspectrum using the previous shift. The different parts of the spectrum that are derived with this process are then assembled into a single graph. The number of Arnoldi steps, $m$, is typically equal to double the number of wanted eigenvalues; larger values of $m$ lead to negligible changes in the values of the calculated eigenvalues. The accuracy of the converged eigenpairs is independently checked by evaluating the residual $|\underline{\underline{J}} \underline{x}-\sigma \underline{\underline{M}} \underline{x}|$, and this quantity is always less than $10^{-10}$ for the reported results.

The linear stability of the viscoelastic stick-slip flow at different $W i$ can be determined from the eigenvalues $\sigma$. For sufficiently small $W i$, all eigenvalues have positive real part, $\operatorname{Re}(\sigma)>0$, so that the corresponding eigenmodes will decay. There is a critical value $W i_{c}$ at which the leading eigenvalue (i.e., the eigenvalue with the minimum real part) crosses the imaginary axis into the left half plane, so that the corresponding eigenmode is linearly unstable. If the corresponding imaginary part, $\operatorname{Im}(\sigma)$, is zero at $W i_{c}$ we have a transcritical or sub(super)critical bifurcation to another steady regular solution. However, if it is not zero then we have a Hopf bifurcation to a time periodic solution. The magnitude of the imaginary part of the crossing eigenvalue corresponds to the temporal oscillation frequency.

The global Jacobian matrix that results after the linearization of the governing equations is stored in Compressed Sparse Row (CSR) format. The implicitly restarted Arnoldi algorithm as it is implemented in the ARPACK library ${ }^{35}$ involves several Gaussian eliminations and for that reason it requires a user-provided solver. As such, we have used PARDISO, a robust, direct, sparse-matrix solver, Schenk and Gärtner. ${ }^{37,38}$ The code is written in Fortran 90 and is run on a workstation with dual Core Xeon CPU at $2.8 \mathrm{GHz}$ in the laboratory of Computational Fluid Dynamics. Each calculation for the stability problem over a wide range of Weissenberg numbers typically requires $2-5$ days to complete, depending on the mesh used.

\section{RESULTS AND DISCUSSION}

In order to validate our code and verify the accuracy of our results, we will first demonstrate that our viscoelastic results converge with mesh refinement. Subsequently we will proceed with a parametric study of our linear stability calculations and we will discuss the possible mechanisms of the instability.

\section{A. Axisymmetric or two-dimensional perturbations $(k=0)$}

\section{Code validation}

In Paper I of this study, ${ }^{24}$ we have examined thoroughly the convergence of the steady solution with mesh refinement. We have shown that for dense enough grids it is possible to resolve well the flow throughout the domain, and especially close to the singularity. We will perform a similar study for the linear stability problem in order to make sure that, in spite of the presence of the singularity at the die exit, the reported results of our stability analysis have converged.

To this end, we have prepared Fig. 1 where the results for the eigenspectrum of a viscoelastic fluid for $\varepsilon=0.05, \beta=0$ are presented for six different meshes, for $W i=2.2$ in the case of a cylindrical die and for $W i=2.8$ in the case of a planar die. Only eigenvalues with positive imaginary part are shown, because eigenvalues appear as complex conjugates. Details for these meshes are presented in Table I. We should note at this point that mesh M8, although it is the grid with the largest number of nodes, it is more evenly refined throughout the domain and not the most refined one close to the 

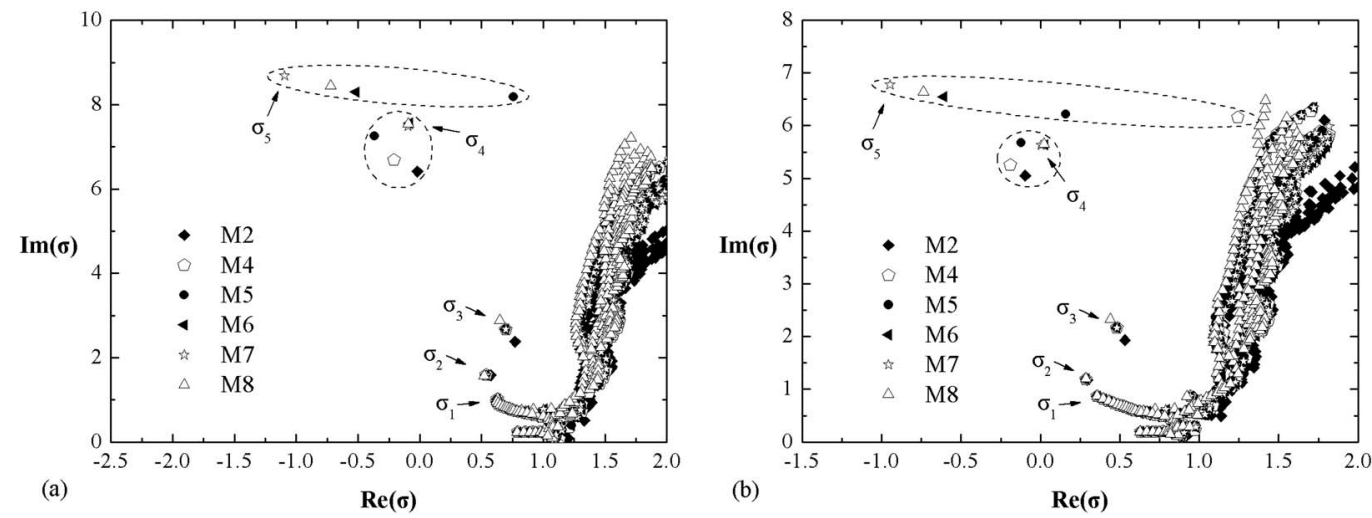

FIG. 1. Effect of the mesh density on the calculated eigenspectrum for $\varepsilon=0.05, \beta=0, l_{1}=10, l_{1}=25$ : (a) cylindrical die $(W i=2.2)$ and (b) planar die $(W i=2.8)$.

exit of the die; the most refined one there is mesh M7, since its extra level of refinement results in elements of smaller size at the die lip (see Table II).

Before proceeding with the discussion of this figure it would be useful first to summarize the characteristics of the spectra of the same or simpler than ours viscoelastic fluids in relevant onedimensional flows. This restriction in the geometry simplifies the stability problem because it allows the linearized equations of motion to be separable, and, hence, reducible to a 1D differential equation in the velocity gradient direction and to examine the spectrum of streamwise wavenumbers, $\alpha$, by assuming the disturbance flow variables to be proportional to $\exp (-\sigma t+i \alpha z)$. The stability of the inertia-less, plane Couette flow of a UCM fluid was examined by Gorodtsov and Leonov. ${ }^{39}$ They proved analytically that for each value of $\alpha$ there exist two discrete eigenmodes, which are now called the "Gorodtsov and Leonov" modes, along with a continuous spectrum. Both types of spectra lie in the stable half-plane. The eigenvalues of the continuous spectrum have real part equal to $1 / W i$ and imaginary part that depends on the wavenumber and the maximum velocity, while, for large $W i$, the real parts of the discrete eigenvalues approach $1 /(2 W i)$ and the imaginary parts approach 0 and $\alpha$. Thus both types of eigenvalues asymptotically approach neutral stability as $W i$ increases. The eigenfunctions corresponding to the discrete spectrum are most dangerous to become unstable, although theoretically they do not. The implications of this behavior on computations using different formulations are discussed extensively and the superiority of the EVSS-G/SUPG method is demonstrated in Ref. 33. In spite of the proximity of the discrete and the continuous eigenspectrum, which makes their numerical resolution particularly difficult, they are enough separated from each other in Couette flow to allow convergence of the critical eigenvalues with mesh refinement. ${ }^{29}$ Renardy and

TABLE I. Properties of typical finite element meshes used in the present work.

\begin{tabular}{|c|c|c|c|c|c|c|c|}
\hline Mesh & $\begin{array}{l}\text { No. of } 1 \mathrm{D} \\
\text { elements in the } \\
(\mathrm{r}, \mathrm{z}) \text { direction }\end{array}$ & $\begin{array}{c}\text { No. of } \\
\text { refinement } \\
\text { levels }\end{array}$ & $\begin{array}{c}\text { No. of } \\
\text { triangular } \\
\text { elements }\end{array}$ & $\begin{array}{c}\text { No. of } \\
\text { unknowns } \\
\text { (base state) }\end{array}$ & $\begin{array}{c}\text { No. of } \\
\text { unknowns } \\
\text { (stability } \\
\text { analysis) }\end{array}$ & $l_{1}$ & $l_{2}$ \\
\hline D1 & $(30,250)$ & 0 & 15000 & 138932 & 216179 & 15 & 35 \\
\hline M1 & $(30,140)$ & 0 & 8400 & 77992 & 121359 & 10 & 25 \\
\hline M2 & $(30,190)$ & 0 & 11400 & 105692 & 164459 & 10 & 25 \\
\hline M3 & $(30,250)$ & 0 & 15000 & 138932 & 216179 & 10 & 25 \\
\hline M4 & $(40,250)$ & 0 & 20000 & 184072 & 286399 & 10 & 25 \\
\hline M5 & $(40,250)$ & 1 & 23427 & 215160 & 334762 & 10 & 25 \\
\hline M6 & $(40,250)$ & 2 & 28180 & 258224 & 401755 & 10 & 25 \\
\hline M7 & $(40,250)$ & 3 & 31316 & 286672 & 446011 & 10 & 25 \\
\hline M8 & $(50,300)$ & 2 & 42420 & 387336 & 602611 & 10 & 25 \\
\hline
\end{tabular}


TABLE II. Convergence of the critical Weissenberg number with mesh refinement for $\varepsilon=0.05$ and $\beta=0$.

\begin{tabular}{lccccc}
\hline \hline & & & & & \\
\cline { 5 - 5 } Mesh & $\Delta r_{\text {min }}$ & $\Delta z_{\text {min }}$ & $\Delta z_{\text {min }} / \Delta r_{\text {min }}$ & Planar die & Cylindrical die \\
\hline M2 & $1.0 \times 10^{-2}$ & $1.0 \times 10^{-2}$ & 0.99 & 2.66 & 2.18 \\
M4 & $7.5 \times 10^{-3}$ & $7.4 \times 10^{-3}$ & 0.98 & 2.52 & 2.03 \\
M5 & $3.7 \times 10^{-3}$ & $3.5 \times 10^{-3}$ & 0.95 & 2.62 & 1.90 \\
M6 & $1.9 \times 10^{-3}$ & $1.7 \times 10^{-3}$ & 0.94 & 2.83 & 2.15 \\
M7 & $9.3 \times 10^{-4}$ & $8.8 \times 10^{-4}$ & 0.95 & 2.80 & 2.14 \\
M8 & $1.5 \times 10^{-3}$ & $1.6 \times 10^{-3}$ & 1.05 & 2.82 & 2.15 \\
\hline \hline
\end{tabular}

Renardy ${ }^{40}$ used a Chebyshev-Tau spectral method to numerically calculate the eigenspectrum of this flow and although they were able to resolve the discrete modes very accurately, the continuous part of the eigenspectrum was insufficiently resolved and appeared in the complex plane as a swollen balloon around a vertical line segment with real part $1 / W i$. This ballooning is attributed to the regular-singular character of the characteristic equation, when the coefficient of its highest derivative vanishes. The real part of the discrete eigenvalues is closer to zero and both types of families of eigenvalues approach each other and the imaginary axis, but never become unstable. The same method was also used by Wilson et al. ${ }^{41}$ to study the stability of the plane Couette and Poiseuille flow, separately or combined, in a channel for a UCM or an Oldroyd-B fluid. They found that in the Couette flow of an Oldroyd-B fluid, a continuous spectrum also exists at the same place as for the UCM fluid and a second continuous spectrum appears with a real part, $1 /(\beta W i)$, i.e., it tends to infinity as $\beta \rightarrow 0$. Moreover the number of discrete modes depends on $\beta$, but its imaginary part still places them symmetrically with respect to the imaginary part of the continuous spectra. Turning to the Poiseuille flow, which is closer to our case, the two continuous spectra are now generated by a branch-cut, but remain inadequately resolved and at the same location. The discrete modes are 6 for the UCM fluid, but their number again depends on $\beta$ for the Oldroyd-B fluid. In the Poiseuille flow the imaginary parts of the discrete eigenvalues no longer place them symmetrically with respect to the imaginary part of the continuous spectrum, because the shear rate is not constant across the channel. Even when the combined Couette and Poiseuille flow is examined, the flow remains stable. The properties of the spectra of the linear stability of the plane Couette and Poiseuille inertialess flows have been further examined by Graham. ${ }^{42}$ He showed that the Gorodtsov and Leonov eigenvalues correspond to eigenvectors that are shear-dominated and localized near the wall. Later on, Grillet et al. ${ }^{28}$ studied the stability of the exponential PTT (ePTT) and the Giesekus models in the planar Couette and Poiseuille flows using either the same spectral method or transient finite element simulations. They gave an expression for the real part of the eigenvalues of the continuous spectrum

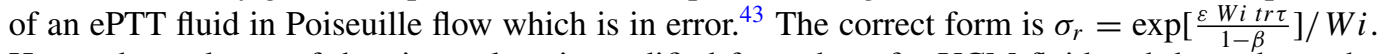
Hence the real part of the eigenvalues is modified from that of a UCM fluid and depends on the solvent viscosity and the $\varepsilon$ parameter of the ePTT model. Generally the shear thinning property and the stress-dependent relaxation time of this fluid model, stabilize the flow because it results in a spectrum that is tilted with respect to the imaginary axis as the imaginary part decreases towards zero. More importantly, they predicted that this flow becomes linearly unstable because a discrete eigenvalue crosses the imaginary axis at a critical $W i$, the value of which depends on $\varepsilon$ with an absolute minimum at $W i \approx 3$.9. Similarly, the ePTT fluid becomes linearly unstable in planar Couette flow, albeit at much larger $W i$, the absolute minimum of which is $W i \approx 45$. Finally, the Giesekus fluid becomes linearly unstable again at fairly large $W i$. Subsequently, Palmer and Phillips ${ }^{44}$ showed that in the planar Poiseuille flow of a linear PTT fluid there also exists a continuous spectrum where the eigenvalues have real part equal to $1 / W i+\varepsilon \operatorname{tr}(\underline{\underline{\tau}}) /(1-\beta)$, as expected considering that the linear PTT model could be obtained in the limit of $\varepsilon \rightarrow 0$ from the exponential one. For the range of parameters investigated in this paper, no instabilities were reported. A new feature of the continuous spectrum was that it "opened up" as the imaginary part approached zero.

Likewise, in our case the spectrum (see Fig. 1) consists of a continuous part located at the same position that is predicted for the Poiseuille flow of a PTT fluid and is not very well resolved, of some 
TABLE III. Dependence of the critical Weissenberg number on the aspect ratio of the mesh elements for $\varepsilon=0.05$ and $\beta=0$, for a cylindrical die.

\begin{tabular}{lcccc}
\hline \hline Mesh & $\Delta r_{\text {min }}$ & $\Delta z_{\text {min }}$ & $\Delta z_{\text {min }} / \Delta r_{\text {min }}$ & $W i_{c r}$ \\
\hline M1 & $1.0 \times 10^{-2}$ & $1.4 \times 10^{-2}$ & 1.4 & 2.20 \\
M2 & $1.0 \times 10^{-2}$ & $1.0 \times 10^{-2}$ & 1.0 & 2.18 \\
M3 & $1.0 \times 10^{-2}$ & $0.7 \times 10^{-2}$ & 0.7 & 2.15 \\
\hline \hline
\end{tabular}

discrete eigenvalues that converge with mesh refinement and of some that do not. In both planar and cylindrical dies the leading eigenmodes, denoted in the figure with $\sigma_{4}$, are discrete and converge with mesh refinement, as found in all studies mentioned above. In addition, we can see in the figure that there is also convergence with mesh refinement for the discrete eigenvalues $\sigma_{1}$ and $\sigma_{2}$. On the contrary, the eigenvalues $\sigma_{3}$ and $\sigma_{5}$ for both dies do not seem to converge with mesh refinement and therefore they will be considered as spurious eigenvalues and will be ignored in our analysis that follows.

Furthermore, in Fig. 1 we observe that the leading eigenvalues for both the cylindrical and the planar die have negative real parts, which means that for this specific Weissenberg number the flow is unstable (see Eq. (4)). As it will be shown below there is a critical value of the $W i$ number, $W i_{c}$, at which the leading eigenvalue crosses the imaginary axis into the left plane so that the flow from stable becomes unstable. In order to show how the calculated values of $W i_{c}$ converge with mesh refinement we have prepared Table II. Clearly, the computed values of $W i_{c}$ converge with mesh refinement for both geometries. Keiller ${ }^{45}$ however, noted that the aspect ratio of the elements can be more important than the overall size of the elements and may lead to spurious eigenvalues. He observed this, while calculating the linear stability of UCM, Oldroyd-B and FENE fluids in planar Couette flow, using a finite difference discretization. Smith et al. ${ }^{29}$ confirmed this finding in their extensive stability studies using the DEVSS-G finite element method. In order to check whether this problem arises in our case we have kept constant the number of the elements in the $r$-direction and changed the number of elements in the $z$-direction (see meshes M1, M2, and M3 in Tables I and III). Although the number of the elements in the axial direction has doubled and the aspect ratio of the elements near the singularity varies from 0.7 to 1.4 the value of $W i_{c}$ is not affected significantly. Finally, we have examined whether the type of triangulation around the singularity affects in any way the calculated spectrum. The triangulation of the mesh which was used for our calculations (see Fig. 2(a) in Ref. 24) changes at the die exit and as a result four triangular elements share one of their nodes at the singular point. Another possibility, which has been used quite often even in similar problems, is the triangulation to remain unchanged inside and outside the die with only three elements sharing their nodes at the die lip. From our numerical experiments it is found that the calculated spectrum is identical in both cases.

To quantify the error of the calculated most critical eigenvector and its dependence on the mesh we have prepared Figure 2. The rate of convergence is evaluated by computing the Euclidean norm of the differences of the values obtained with any mesh, Mn, to those obtained by the finest mesh (M7) for the same variables. This has been performed for a cylindrical die for the cross-section at the die exit $\left(0 \leq r \leq 1, z=l_{1}\right)$ and are presented in Fig. 2. For the computation of the norms the values of all variables at meshes other than the coarsest one are interpolated at the locations corresponding to the coarsest mesh. Clearly for all the variables the relative error decreases quadratically with mesh refinement. The picture is similar for a cylindrical die as well. In all cases to be presented subsequently the M4 mesh is used, unless otherwise noted.

Apart from the mesh refinement study, we should also examine the effect of the location of the inflow and outflow boundaries or even the type of boundary condition that is applied therein. As we mentioned earlier the flow at these boundaries is considered to be fully developed. This consideration, however, is subject to verification since these boundaries are located at a finite distance from the die and therefore it is expected that these conditions may introduce some spurious eigenmodes. ${ }^{25}$ As we can see in Fig. 3, for a cylindrical die, placing the inflow and outflow boundaries, respectively, 5 and 

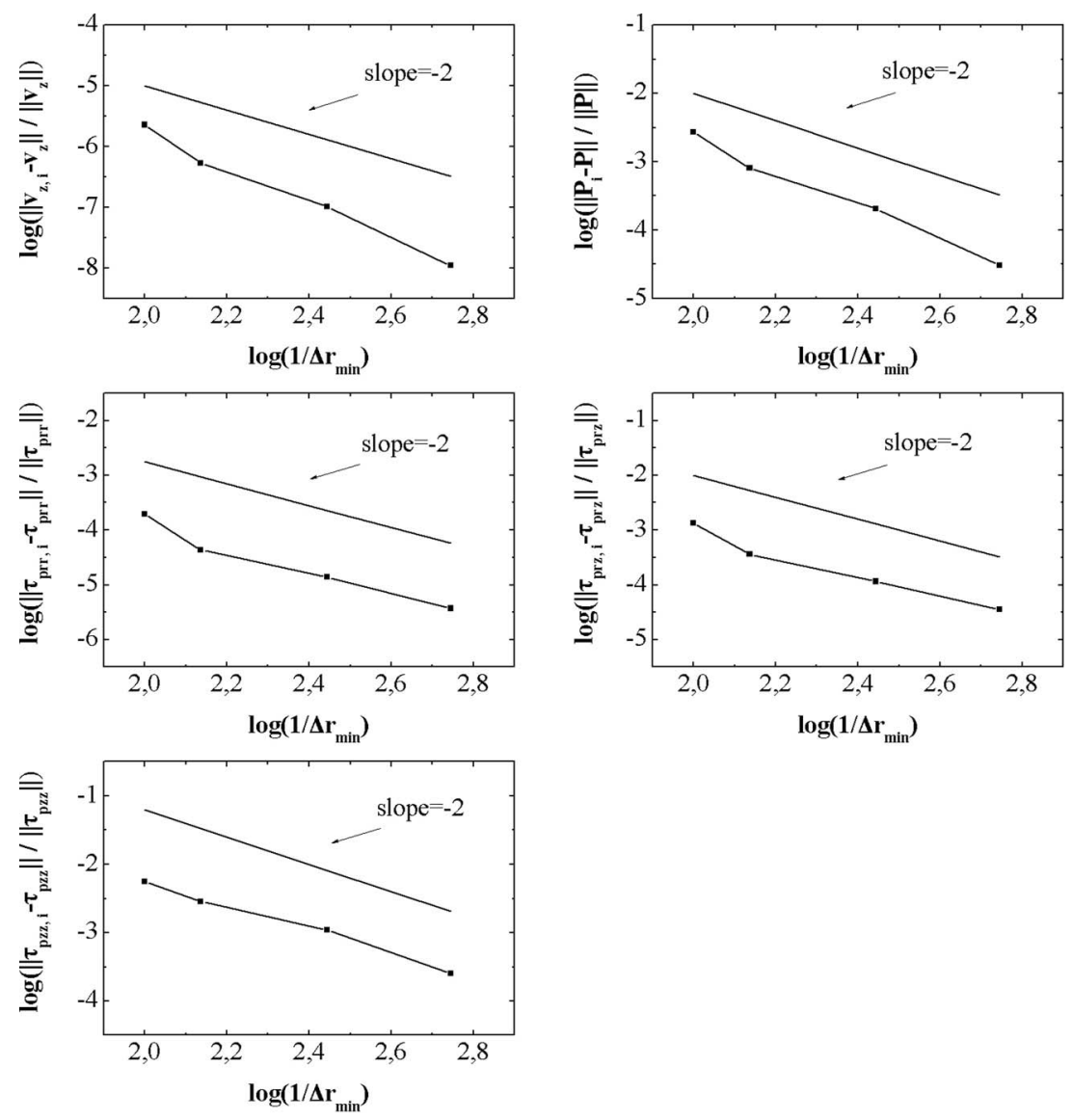

FIG. 2. The relative error of the pressure and the stresses disturbances at the exit of a planar die $(z=10)$ for $W i=2.8$, $\varepsilon=0.05, \beta=0, l_{1}=10, l_{2}=25$, and various meshes. As a reference value the solution with the mesh M7 is used, while the vectors $P_{i}^{\prime}, \Sigma_{r r, i}^{\prime}, \Sigma_{r z, i}^{\prime}, \Sigma_{z z, i}^{\prime}$ are calculated using the meshes M2, M4, M5, and M6.

10 half gap widths further away from the die exit has no effect on the discrete part of the spectrum, which remains the stability determining part. We get a very similar picture for the case of a planar die.

Although the leading eigenvalues are not affected by the location of the inflow and outflow boundaries we think that it would be important to study the possible effect of the type of boundary condition that is applied at the outflow boundary. Therefore we have applied alternative boundary conditions, all implying fully developed flow, and checked their effect on the calculated eigenvalues. In Table IV we present the calculated eigenvalues using (a) Eq. (14) in Ref. 24, i.e., boundary conditions on velocities $v_{r}=0, \frac{\partial v_{z}}{\partial z}=0$, (b) boundary conditions on stresses $\tau_{r z}=0, \tau_{z z}=0$, and (c) the open boundary condition (OBC) that has been proposed by Papanastasiou et al. ${ }^{46}$ According to it the surface integrals of the momentum equations are evaluated in terms of the, as yet, unknown nodal values of velocity, pressure and stress and added to the volume integrals of the corresponding elements. As it can be seen, although the imposed boundary conditions differ significantly, the calculated leading eigenvalues are not affected at all. Concluding, we find that the leading eigenvalues are not affected either by the mesh or the type or the location of the inflow and outflow boundaries and therefore they can be used for determining the stability of the flow.

Finally, we performed a final test in order to assure that our computations do not produce nonphysical results. It is well known that the conformation tensor that corresponds to the calculated stress field should always be positive definite, otherwise the viscoelastic computations have no physical 


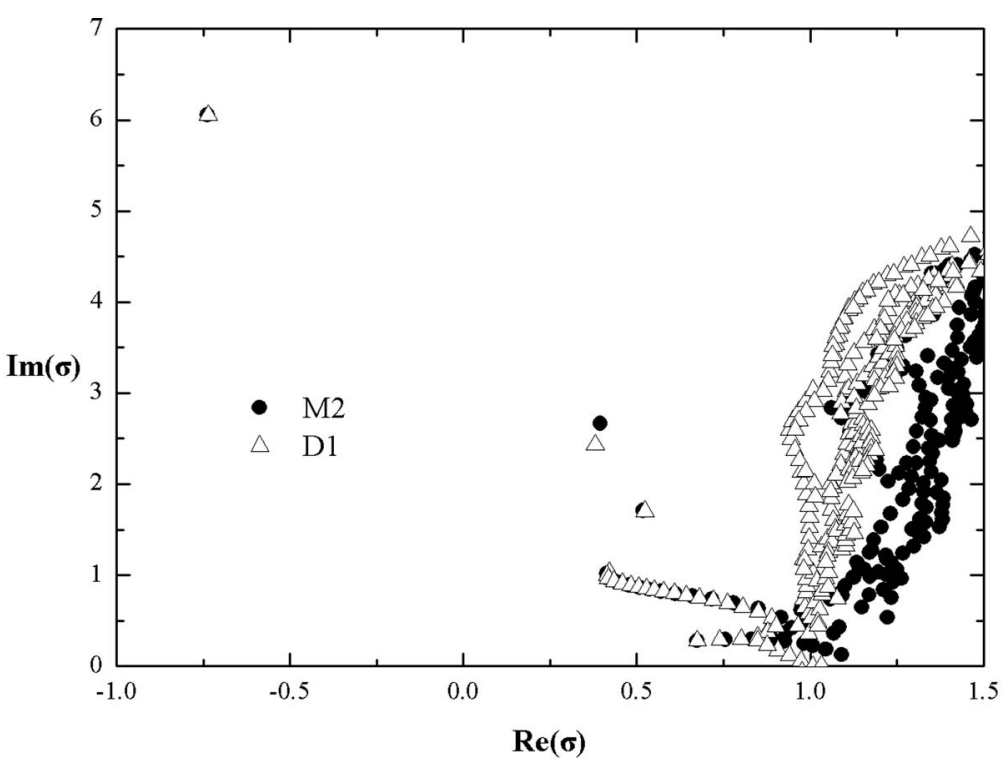

FIG. 3. Effect of the location of the inflow and outflow boundaries on the calculated eigenspectrum for $W i=2.5, \varepsilon=0.05$, $\beta=0$, using mesh M2 $\left(l_{1}=10, l_{2}=25\right)$ and D1 $\left(l_{1}=15, l_{2}=35\right)$ : Cylindrical die.

meaning. The conformation tensor is related to the stress tensor using the following equation:

$$
\stackrel{\tau}{=} p=\frac{1-\beta}{W i}(\underline{\underline{c}}-\underline{\underline{I}}) \text {. }
$$

We have calculated the conformation tensor part of the steady state solution and the eigenvector for various $W i$ near criticality for the stick-slip flow of a PTT fluid and found that in all cases the conformation tensor remains positive definite.

Therefore, we have verified the convergence with mesh refinement using differently structured meshes and assured that the calculated spectrum is not affected by the inflow and outflow boundary conditions. Moreover, the unstable mode is discrete from the continuous portion of the spectrum. Hence we believe that the instability calculated here is an accurate reflection of the dynamics of the ePTT model in stick-slip flow and not a numerical artifact.

\section{Effect of material properties on critical conditions for instability}

Now we may proceed with the parametric study to examine the effect of various rheological properties of the material on the stability of the stick-slip flow. The dependence of the eigenvalue spectrum on the Weissenberg number is shown in Fig. 4 for a cylindrical die and for $\varepsilon=0.05$, $\beta=0$. We can see that as the Weissenberg number increases from low values (see Fig. 4(a)), the cluster of the eigenvalues for both geometries moves towards the imaginary axis. In all cases, its real part varies from approximately $1 / W i$ for nearly zero imaginary part to higher values of both its real and imaginary parts. The discrete eigenvalues are located closer to the imaginary axis with respect to the continuous part, i.e., these modes are less stable. As we have already mentioned, Wilson et al. ${ }^{41}$

TABLE IV. Value of the leading eigenvalue using three different outflow boundary conditions for $W i=3, \varepsilon=0.05$, and $\beta=0$ with mesh M6.

\begin{tabular}{lccc}
\hline \hline & $v_{r}=0, \frac{\partial v_{z}}{\partial z}=0$ & $\tau_{r z}=0, \tau_{z z}=0$ & OBC \\
\hline Planar die & $-0.1521+5.4852 \mathrm{i}$ & $-0.1521+5.4853 \mathrm{i}$ & $-0.1520+5.4852 \mathrm{i}$ \\
Cylindrical die & $-0.9649+6.6513 \mathrm{i}$ & $-0.9648+6.6511 \mathrm{i}$ & $-0.9649+6.6514 \mathrm{i}$ \\
\hline \hline
\end{tabular}





FIG. 4. Effect of viscoelasticity on the eigenvalues calculated with mesh M4 for a cylindrical die and $\varepsilon=0.05, \beta=0$, $l_{1}=10, l_{2}=25$. (a) Low $W i$ numbers and (b) Wi near criticality.

proved that in the planar Poiseuille flow of a UCM fluid a continuous spectrum exists where the eigenvalues have real part equal to $1 / W i$, whereas Grillet et $a l^{28}$ and Palmer and Phillips ${ }^{44}$ showed that in the case of an exponential or a linear PTT fluid this dependence is affected by the $\varepsilon$ and the $\beta$ parameters of these fluid models. We find that this modified dependence is in accordance with our computations for larger values of $W i$ as it is shown in Fig. 4(b). We observe that the majority of the clustered eigenvalues move slower than $1 / W i$ towards the imaginary axis and this is due to the effect of shear thinning. A second cluster of eigenvalues is not found because it is displaced towards infinity, given that $\beta=0$ in Figure 4 or remains quite small in subsequent figures. However, we found that the leading eigenvalues of those move faster towards the imaginary axis while, for some value of $W i$, they cross it indicating a transition to instability. The picture is very similar for the case of a planar die.

A detailed calculation shows that the crossing occurs at $W i_{c}=2.03$ for the cylindrical die and at $W i_{c}=2.52$ for the planar one (calculated with the M4 mesh). Moreover these eigenvalues are complex which means that we have a Hopf bifurcation and the flow that results is periodic in time. We should note here that this finding is in agreement with the work of Venet and Vergnes. ${ }^{14}$ These authors showed detailed profilometric and microscopy data of the observed instability indicating that, above a certain value of the shear stress, the surface defect that arises first is periodic with a quickly increasing amplitude suggesting the presence of a Hopf bifurcation. The values of the critical Weissenberg numbers for the cylindrical geometry are pretty close to the critical recoverable shear, $S_{R}=1.5$, seen in the experiments performed by Kalika and Denn ${ }^{9}$ or the value $S_{R}=1.73$ which was given later by Pomar et al. ${ }^{13}$ We should note that the recoverable shear is defined as $S_{R}=\frac{\tau_{w}}{G}$, where $\tau_{w}$ is the wall shear stress and $G$ is the elastic modulus. The latter can be obtained from $G \approx \frac{\mu}{\lambda}$, while the wall shear stress is $\tau_{w} \approx \frac{\mu U}{R}$. Therefore it follows that the recoverable shear is roughly equal to Weissenberg number, $S_{R} \approx W i$, and the experimentally obtained critical value 


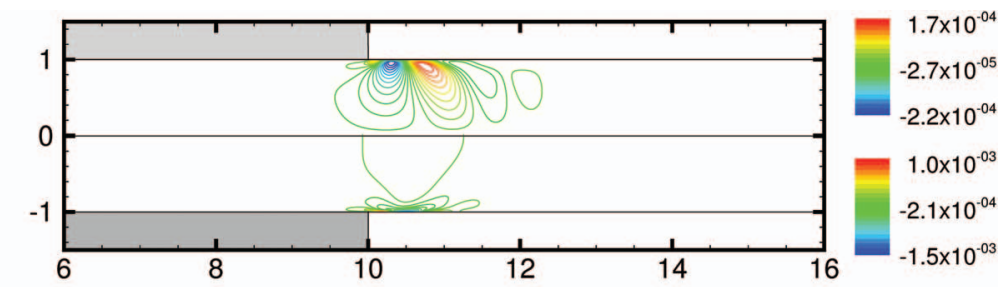

(a)

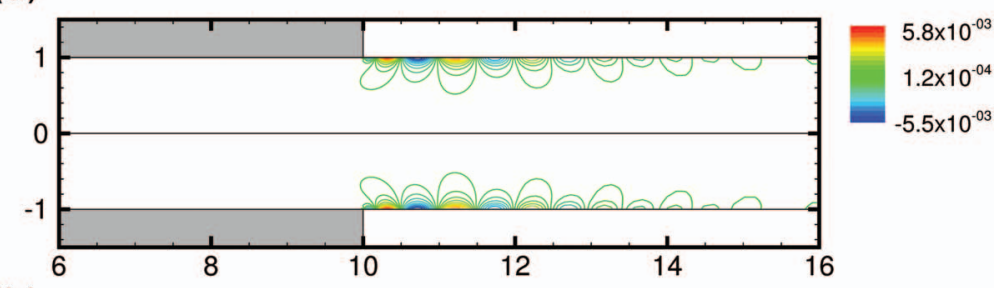

(b)

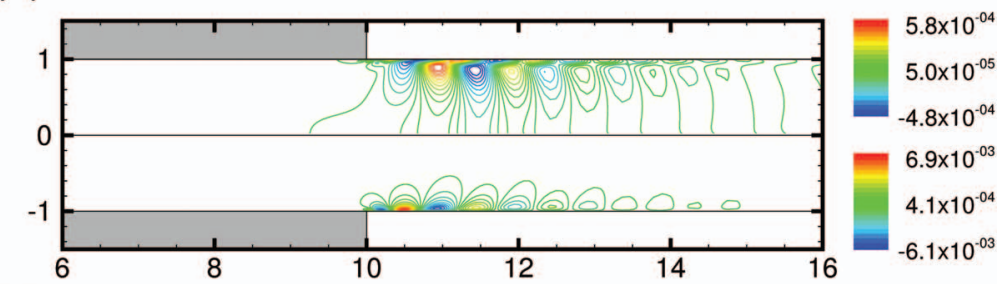

(c)

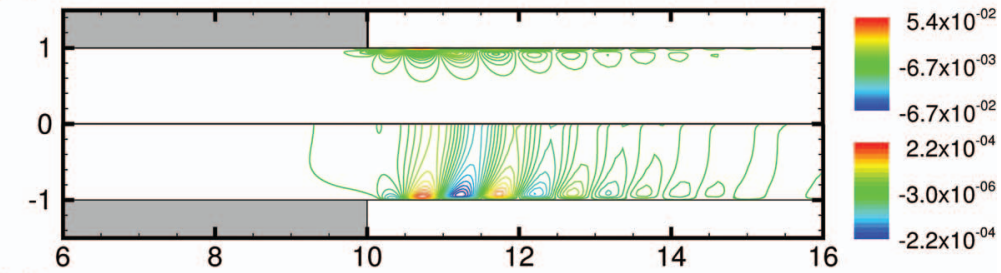

(d)

FIG. 5. Spatial form of the eigenvector for a cylindrical die. Isolines of (a) $v_{r}^{\prime}, v_{z}^{\prime}$, (b) $P^{\prime}$, (c) $\tau_{p r r}^{\prime}, \tau_{p r z}^{\prime}$, and (d) $\tau_{p z z}^{\prime}, \tau_{p \theta \theta}^{\prime}$ on the upper and lower half respectively for $W i=2.1, \varepsilon=0.05, \beta=0, l_{1}=10, l_{2}=25$ (for clarity we show the region $5 \leq z \leq 16)$. The corresponding eigenvalue is $\sigma=-0.0928+6.7613 i$. Mesh M4 is used.

for $S_{R}$ is close to our computed critical $W i$. The agreement is striking taking into consideration the fact that in this work we only examine the stick-slip flow and do not take into account the effect of the free surface as we should in the full extrudate swell problem.

The spatial variation of the most unstable eigenvector is illustrated in Figs. 5 and 6 for the cylindrical and the planar die, respectively. We can see that the perturbations of the velocity and stress field initiate at the die exit but they are primarily concentrated outside the die and on the surface of the extrudate. No variation is observed in the entrance or well before the exit of the die, in agreement with experiments reporting that the entrance conditions do not influence the sharkskin instability, ${ }^{14}$ while sharkskin is a die-exit phenomenon. ${ }^{12,18,47}$ Besides the fact that the flow in the unstable regime is periodic in time, since the corresponding eigenvalue is complex, it is obvious that the perturbation also has a spatial periodic structure especially for the perturbations of the pressure and the stress field the values of which have alternating signs along the slip surface. The oscillation of all variables follows the same periodic structure in space. Among the polymeric stress components, the oscillation is most intense for the axial normal stress and the least intense for the radial normal stress in direct correspondence to the magnitudes of the stress components in the base state. ${ }^{25}$ The hoop stress, although it assumes intermediate values, seems to intensify the instability. This could be the reason that the critical Weissenberg number is consistently lower in the cylindrical than that in the planar geometry. 


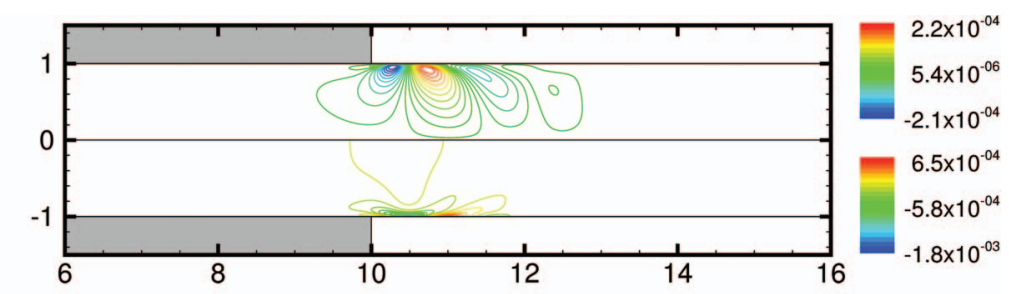

(a)

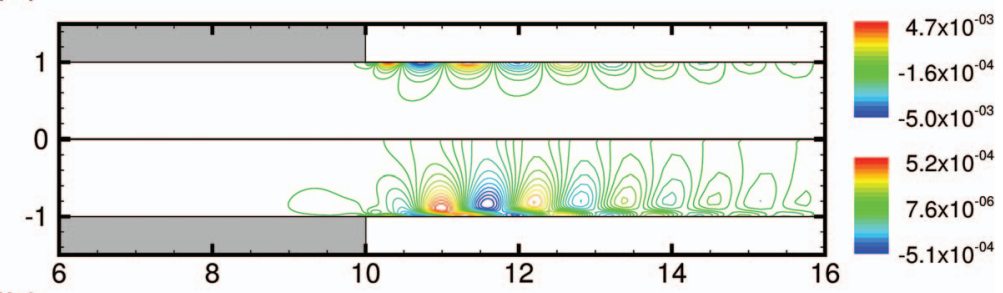

(b)

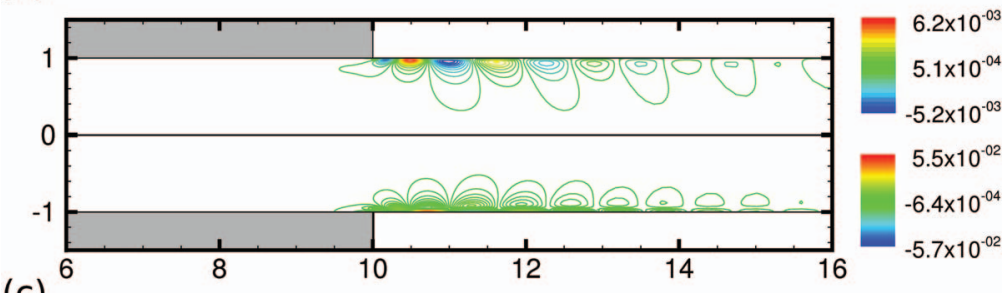

FIG. 6. Spatial form of the eigenvector for a planar die. Isolines of (a) $v_{r}^{\prime}, v_{z}^{\prime}$, (b) $P^{\prime}, \tau_{p r r}^{\prime}$, and (c) $\tau_{p r z}^{\prime}, \tau_{p z z}^{\prime}$ on the upper and lower half respectively for $W i=2.6, \varepsilon=0.05, \beta=0, l_{1}=10, l_{2}=25$ (for clarity we show the region $5 \leq z \leq 16$ ). The corresponding eigenvalue is $\sigma=-0.0507+5.3677 i$. Mesh M4 is used.

The amplitudes are highest right at the die exit, but decrease downstream. Apparently the stresses that develop at the die $\operatorname{lip}^{25}$ beyond critical conditions are sufficiently high. Especially the larger axial normal stress causes abrupt elongation of the polymer chains and more so in its surface than its core, where in fact the polymer is axially compressed. This intense surface elongation may exceed a critical value causing the highly entangled polymer to respond like a solid, which ruptures producing "cracks" at the surface of the material as it leaves the die. The "cracks" should appear right at the outlet of the die and temporarily relax the high stresses, which build up again until the next "crack". The "cracks" could close downstream because of viscous dissipation and material relaxation. This variation has been observed often in extrusion experiments ran in the sharkskin parameter range. ${ }^{10,12}$ This scenario leading to sharkskin was first suggested by Cogswell, ${ }^{15}$ while the sharp increase of the stresses near the die lip was confirmed by birefringence experiments. ${ }^{12,48,49}$ If we extend these results to the more general extrudate swell problem, it would be reasonable to expect that this dynamic flow field could induce a wavy free surface which is characteristic of the instabilities which appear in the experiments. We observe that for the cylindrical case the wave length of the perturbation is about 0.5 of the extrudate diameter. From the experiments carried out by Kalika and Denn ${ }^{8}$ the wave length is about 0.23 diameters, which is again close enough given that here we examine the stick-slip problem.

Another interesting experimental finding ${ }^{14}$ is that, the amplitude and the period of the surface defects increase with the wall shear stress just above the critical conditions for the sharkskin instability. The former cannot be examined in the context of the linear stability analysis. The latter is examined in Fig. 7, where we compare, for example, the part of the eigenvector corresponding to the azimuthal normal stress either for $W i=2.1$ on the upper or for $W i=3$ on the lower half of the figure. Clearly, the spatial period of the disturbance increases with the Weissenberg number. The spatial period is inversely proportional to the temporal frequency of the sharkskin instability, i.e., the imaginary part of the corresponding eigenvalue. So we can quantify this dependence more easily by plotting the imaginary part of the eigenvalue as a function of Wi near criticality. Fig. 8 


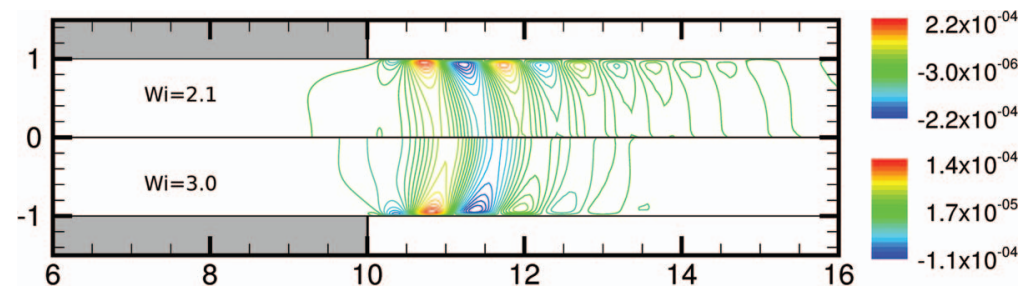

FIG. 7. Spatial form of the eigenvector of the most unstable mode for a cylindrical die. Isolines of $\tau_{p \theta \theta}^{\prime}$ for $W i=2.1$ on the upper and for $W i=3$ on the lower half. The rest of the parameters are $\varepsilon=0.05, \beta=0, l_{1}=10, l_{2}=25$ (for clarity we show the region $6 \leq z \leq 16$ ). The corresponding eigenvalues are $\sigma=-0.0928+6.7613 i$ for $W i=2.1$ and $\sigma=-0.8815$ $+6.2228 i$ for $W i=3.0$. Mesh M4 is used.

demonstrates that indeed the temporal frequency decreases with $W i$ for both geometries. Hence we have another qualitative agreement with experiments, in spite the simplified geometry examined.

In order to investigate the parameters that affect this instability it would be interesting to examine the effect of the various rheological parameters of our viscoelastic model. First we will examine the effect of the parameter $\varepsilon$. This parameter introduces to the PTT model the effect of shear thinning as well as an extensional viscosity which is varying with extensional hardening followed by extensional thinning for smaller values of $\varepsilon$ or extensional thinning only for larger values. It is expected that the effects of shear and elongational thinning become increasingly important as we approach the triple contact point where the stresses become very high and therefore may affect significantly the stability of the flow. The dependence of the shear and elongational viscosity on the shear or elongation rate respectively is presented in Fig. 9. Clearly, the smaller the value of $\varepsilon$ the more intense the elongational thickening that the fluid experiences while for $\varepsilon=0$ (UCM fluid) the extensional viscosity is unbounded. For the latter fluid the shear viscosity is constant for all shear rates, whereas the increase of the parameter $\varepsilon$ introduces shear thinning in lower and lower shear rates.

Now that we have seen the effects of the parameter $\varepsilon$ on the shear and elongational properties of the viscoelastic fluid we may examine its effect on the flow stability. To this end, we present the calculated critical Weissenberg number as a function of the parameter $\varepsilon$ in Fig. 10. As $\varepsilon$ tends to zero and the PTT model tends to the UCM limit the value of $W i_{c}$ increases rapidly. This is in accord with all previous studies demonstrating that the UCM fluid in Couette and Poiseuille flow is always

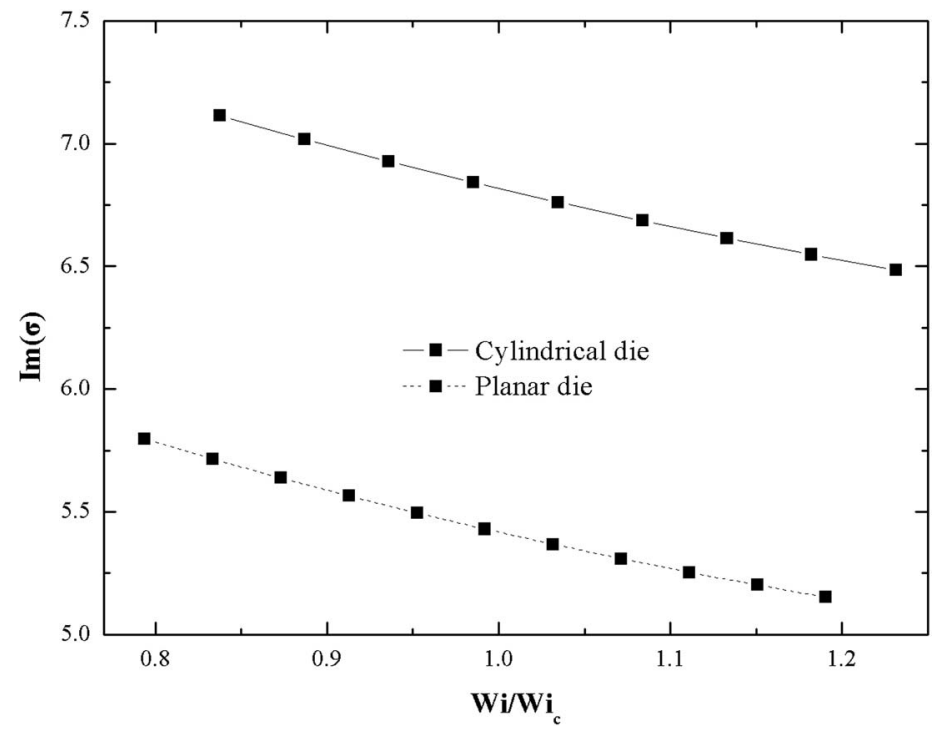

FIG. 8. Dependence of the imaginary part of the most dangerous eigenvalue on the Weissenberg number for $\varepsilon=0.05$, $\beta=0, l_{1}=10, l_{2}=25$. Mesh M4 is used. 


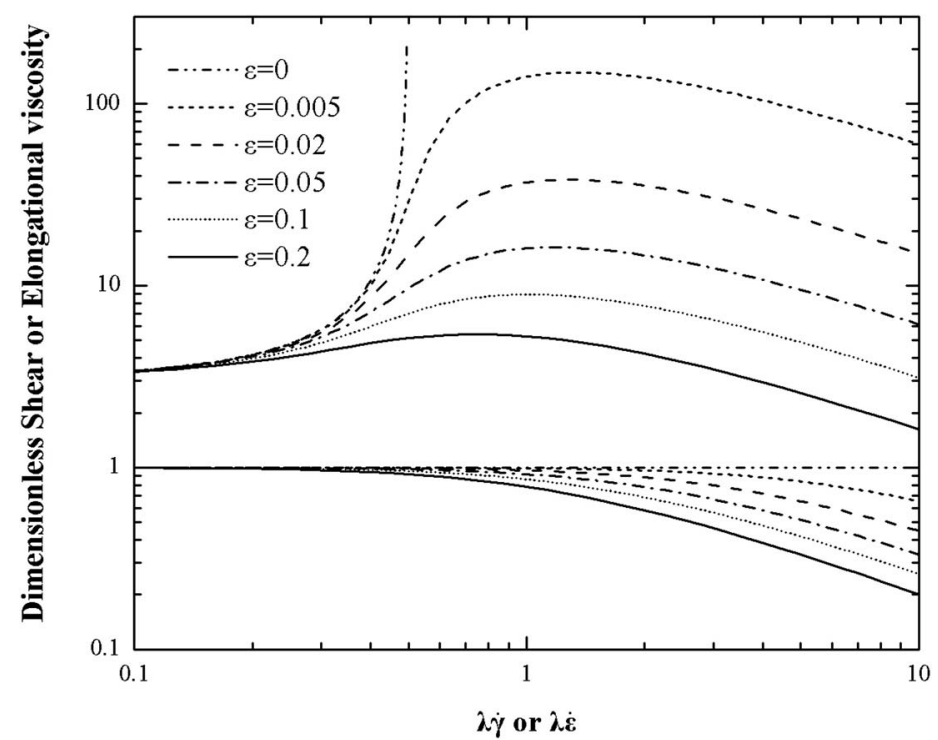

FIG. 9. Shear and elongational properties for a PTT fluid with $\beta=0$.

stable. ${ }^{39-41}$ It appears that strain thinning, which is introduced to the model through the parameter $\varepsilon$, is a destabilizing factor for the stick-slip flow of a viscoelastic fluid. Increasing the value of $\varepsilon$ the critical Weissenberg number decreases abruptly at small values of $\varepsilon$ and reaches a minimum for $\varepsilon \sim 0.06$ in both geometries. Further increase of this parameter, which corresponds to a highly shear thinning material, leads to stabilization of the flow. A similar behavior with $\varepsilon$ has also been reported by Grillet $e t a l .{ }^{28}$ for the stability of Poiseuille flow of a PTT fluid, albeit we have to note that the most unstable mode in their case is not the same with ours. Venet and Vergnes ${ }^{14}$ in their experiments have observed that polymers exhibiting long chain branching and more strain hardening are less sensitive to sharkskin. This concurs with our prediction of the dependence of $W i_{c}$ on $\varepsilon$ for strongly strain hardening materials, $\varepsilon<0.05$, see Fig. 9.

Next, we examine the effect of the solvent by depicting in Fig. 11 the dependence of $W i_{c}$ on the solvent viscosity ratio, $\beta$. It is shown that the solvent has a strongly stabilizing effect. This is hardly surprising since one would expect that the addition of a Newtonian solvent would result in a more

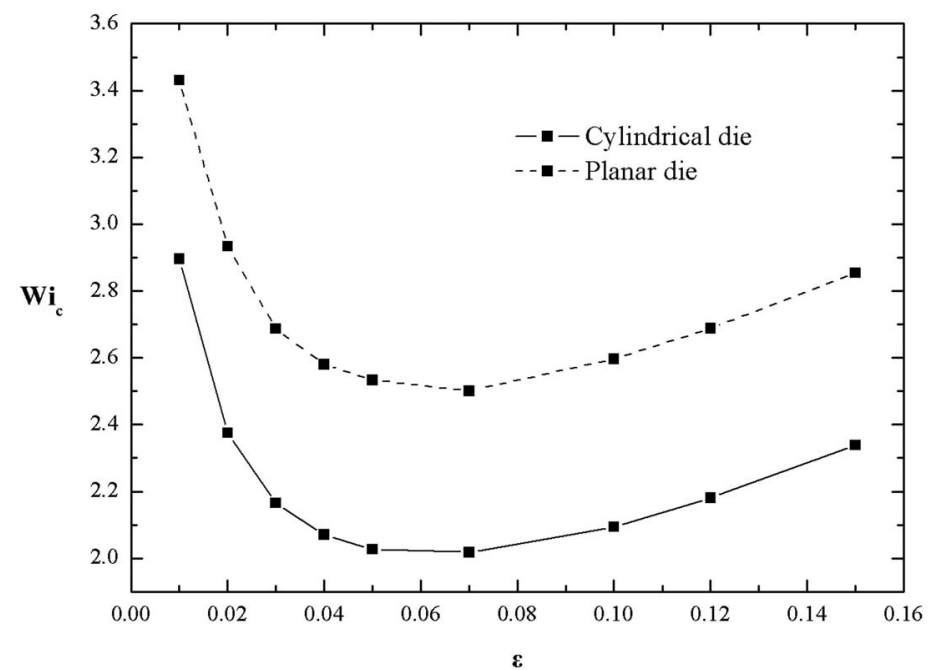

FIG. 10. Critical Weissenberg number as a function of the extensional parameter, $\varepsilon$, for $\beta=0$. Mesh M4 is used. 




FIG. 11. Critical Weissenberg number as a function of the solvent viscosity ratio, $\beta$, for $\varepsilon=0.05$. Mesh M4 is used.

Newtonian-like behaviour. Further increase of $\beta$ was not pursued because the focus of this study is the case of polymer melts. Furthermore, in Paper I of this study ${ }^{24}$ it was noted that the singularity becomes more intense with increase of $\beta$ and therefore it is expected that the convergence of the eigenvalues may deteriorate.

It would be interesting to see the effect that the above model parameters have on the temporal frequency of the dynamic flow in the unstable regime. To this end we have presented in Fig. 12 the dependence of the imaginary part of the leading eigenvalue, computed at $W i_{c}$, on the extensional parameter $\varepsilon$ for $\beta=0$. As we can see the imaginary part increases with $\varepsilon$, which means that the temporal frequency increases as strain thinning becomes more important. In addition we present in Fig. 13 the dependence of the imaginary part of the leading eigenvalue, computed at $W i_{c}$, as a function of the solvent viscosity ratio. From the figure we deduce that the temporal frequency decreases as the Newtonian solvent contribution increases.

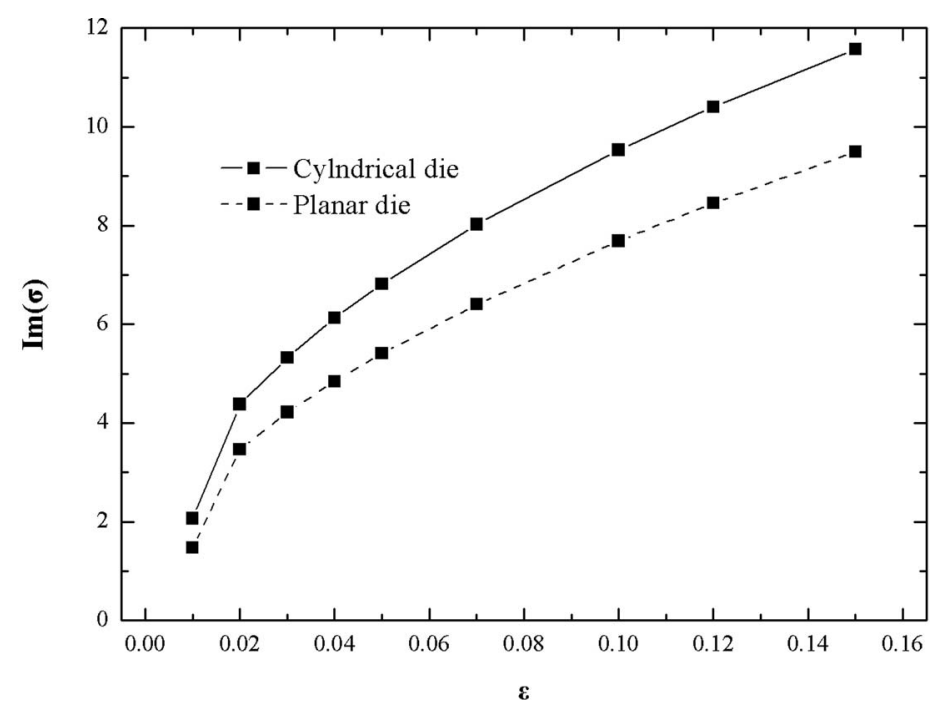

FIG. 12. Effect of the extensional parameter, $\varepsilon$, on the imaginary part of the leading eigenvalue computed at $W i_{c}$ for $\beta=0$. Mesh M4 is used. 


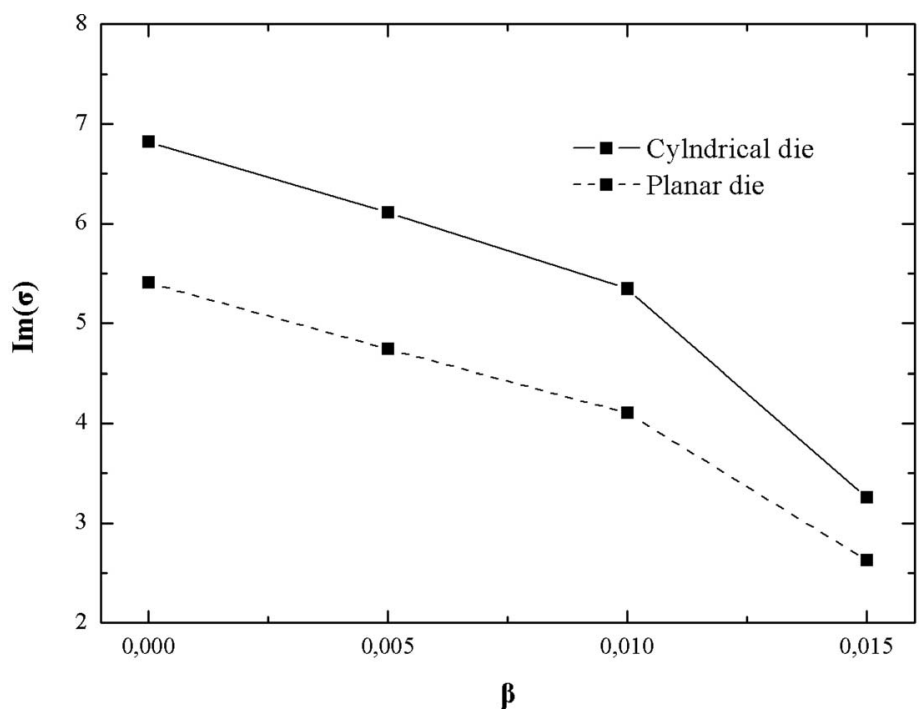

FIG. 13. Effect of the solvent viscosity ratio, $\beta$, on the imaginary part of the leading eigenvalue computed at $W i_{c}$ for $\varepsilon=0.05$. Mesh M4 is used.

\section{Examination of the next to the critical eigenvalue}

Although in order to study the stability of a flow one only has to follow the most unstable eigenvalue, it would be interesting to see the form of the eigenvector which corresponds to the next to critical discrete eigenvalue, which, could become unstable at a higher Weissenberg number. Indeed, this occurs at values somewhat less than $W i_{c} \approx 5.6$ for a cylindrical die and $W i_{c} \approx 5.4$ for a planar die, when $\varepsilon=0.05, \beta=0$. We have depicted the spatial variation of this eigenvector in Fig. 14 for a cylindrical die. Now the perturbations of the velocity and stress field are primarily concentrated inside the die, while their values outside the die are one order of magnitude (or more) smaller than those inside it. Again we observe that the perturbation has a spatially periodic structure as in Figs. 5 and 6 and that the eigenvectors vary more near the stationary wall as explained in Ref. 33. In fact, a boundary layer in $v_{z}$ and $\tau_{p z z}$ is formed near the wall. Moreover we observe that the velocity boundary layer thickness is larger than the one for the stress which is in agreement with the theoretical analysis given by Black and Graham ${ }^{23}$ who considered the PTT viscoelastic model together with a slip model which includes a dependence of the slip velocity not only on shear stress but also on normal stress.

We also note that, as in the case of the most critical eigenvalue, the perturbations seem to be localized close to the die exit. To make sure that the eigenvectors of the next to critical eigenvalue are not affected in any way by the position of the inlet boundary we performed simulations varying the length of the inlet domain, shown in Fig. 15; clearly, the position of the inlet boundary perturbations does not play any significant role. The perturbations are initiated in the vicinity of the die lip and the amplitude of the oscillations decreases moving upstream. The fact that the perturbations are localised at the die exit implies that, despite the similarities, this mode must be different from the mode that was reported to be most unstable in the Poiseuille flow of an ePTT fluid by Grillet et $_{\text {al. }}{ }^{28}$

At this point, we should note that an oscillatory and spatially periodic flow, without, however, the presence of boundary layers in the stress field, was also reported by Brasseur et al.,${ }^{17}$ who simulated the time-dependent axisymmetric stick-slip and die swell flow of an OLDROYD-B fluid following a non linear slip law along the wall. They have suggested that the combination of viscoelasticity and non-monotonic slip at the die walls could lead to this oscillatory flow. Moreover, they have shown for the die swell problem that this periodic flow also generates small amplitude waves on the surface of the extrudate. It is striking that, although our model does not include any wall slip, it is able to predict a similar form of instability. We must note, however, that the PTT model, in contrast to the 

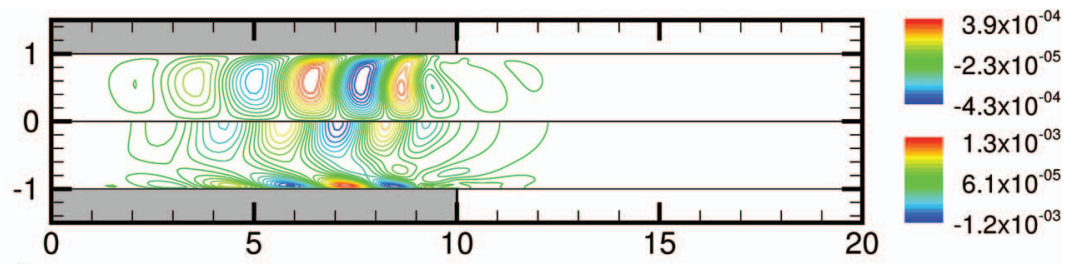

(a)

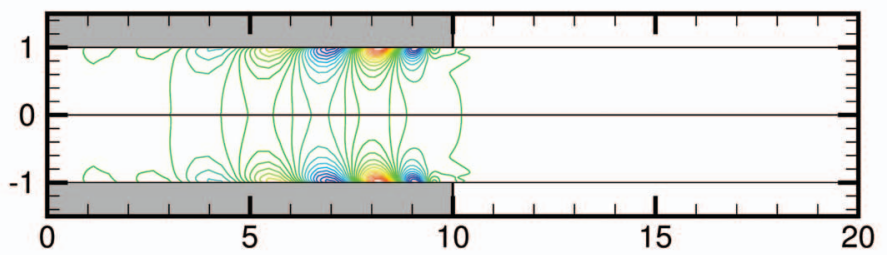

$6.3 \times 10^{-03}$

$3.5 \times 10^{-04}$

$-5.6 \times 10^{-03}$

(b)
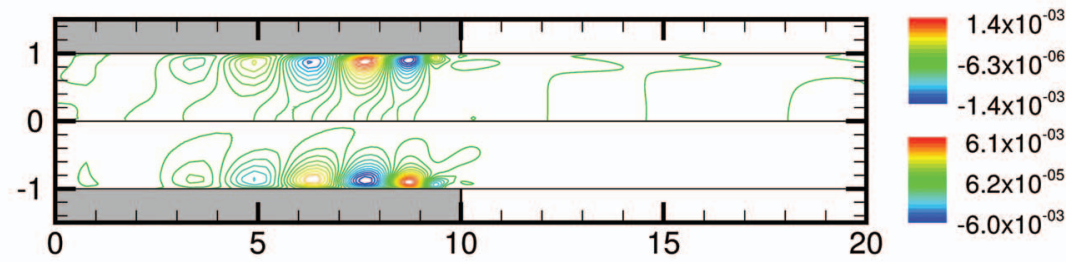

(c)
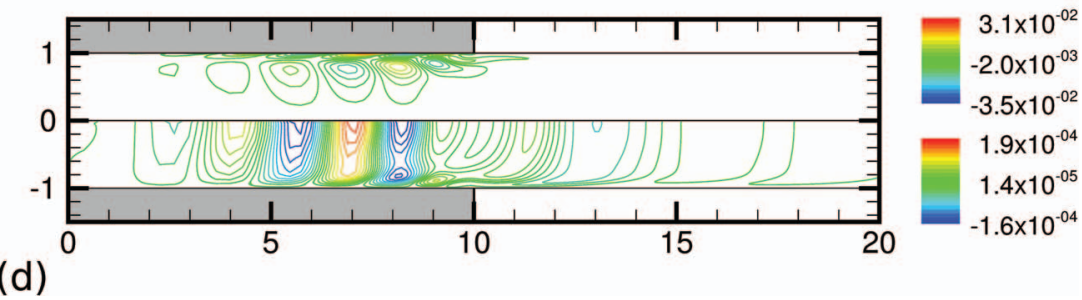

(d)

FIG. 14. Spatial form of the eigenvector for a cylindrical die. Isolines of (a) $v_{r}^{\prime}, v_{z}^{\prime}$, (b) $P^{\prime}$, (c) $\tau_{p r r}^{\prime}, \tau_{p r z}^{\prime}$, and (d) $\tau_{p z z}^{\prime}, \tau_{p \theta \theta}^{\prime}$ on the upper and lower half respectively for $W i=5.6, \varepsilon=0.05, \beta=0, l_{1}=10, l_{2}=25$ (for clarity we show the region $0 \leq z \leq 20$ ). The corresponding eigenvalue is the second most critical and its value is $\sigma=-0.0008+1.0422 i$. Mesh M4 is used.

OLDROYD-B model used by Brasseur et al. ${ }^{17}$ predicts a varying shear and elongational viscosity and our simulations still employ the usual no-slip boundary condition. Consequently it is reasonable to assume that the essential features of a constitutive model in order to predict instability which qualitatively agrees with experimental findings are a combination of viscoelasticity and elongational thinning whereas a separate slip law is not necessary.

\section{B. Energy analysis}

In order to identify the physical mechanism leading to instability we perform an "energy budget" analysis which has been used with success in the past for the analysis of various viscoelastic flows. ${ }^{28,50-54}$ The energy method considers the interaction of the base flow and the disturbance flow by evaluating the mechanical energy balance for the perturbed system. ${ }^{55}$ Hence it is used to determine the stabilizing and destabilizing effects of the coupling of velocities and stresses from the base flow and perturbation flow. The methodology that will be described below is similar to the approach of Joo and Shaqfeh ${ }^{51}$ and Ganpule and Khomami ${ }^{53}$ who introduced it for an Oldroyd-B fluid model.

The disturbance energy equation is obtained by taking the inner product of the linearized perturbation of the momentum equation with the perturbation velocity and integrating the resulting 
(a)
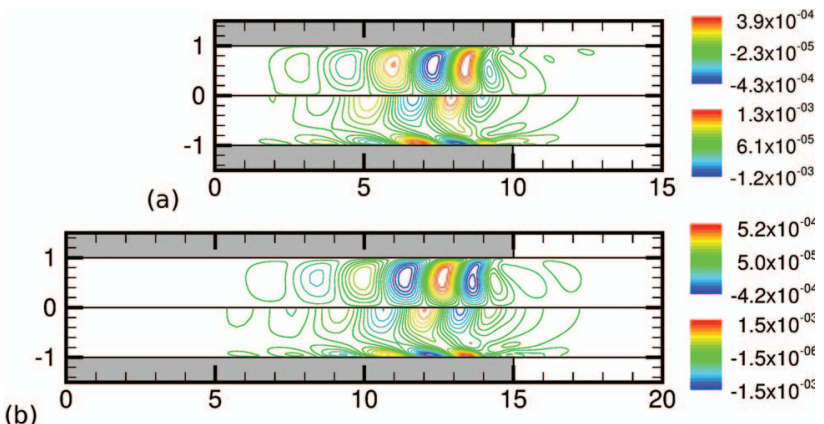

FIG. 15. Spatial form of the eigenvector that corresponds to the next to the critical eigenvalue for a cylindrical die. Isolines of $v_{r}$ and $v_{z}$, on the upper and lower half, respectively, for $W i=5.6, \varepsilon=0.05, \beta=0$ and for (a) $l_{1}=10$ (Mesh M2) and (b) $l_{1}=15$ (Mesh D1).

equation over the volume of the flow field and one periodic cycle in time (i.e., $0<t<2 \pi / \sigma_{I}$ ).

$$
\int\left[\nabla \hat{P}-\beta \nabla \cdot\left(\underline{\underline{\hat{G}}}+\underline{\underline{\hat{G}}}^{T}\right)-\nabla \cdot \underline{\underline{\hat{\tau}}}_{p}\right] \cdot \underline{\underline{\hat{v}}} d \bar{V}=0
$$

where this notation implies the volume and time integrals of a quantity A, i.e. $\int \mathrm{A} d \bar{V}$ $=\int_{0}^{2 \pi / \sigma_{I}} \int_{V} \mathrm{~A} d V d t$. Note that for the evaluation of all terms shown in Eq. (12) and in the equations below we take just the real part of the perturbations, $\underline{\hat{v}}, \hat{P}, \underline{\hat{G}}$, and $\underline{\underline{\hat{\Sigma}}}$.

After some manipulation (see the Appendix for a detailed derivation) the energy budget becomes

$$
\frac{d V D}{d t}=\varphi_{p r}-\varphi_{v i s}+\varphi_{\text {relax }}+\varphi_{p v 1}+\varphi_{p v 2}+\varphi_{p s 1}+\varphi_{p s 2}-\varphi_{e l}+\varphi_{j u m p},
$$

where

$$
\frac{d V D}{d t}=\int W i \frac{1-\beta}{Y_{b}} \frac{\partial}{\partial t}\left(\underline{\underline{\hat{G}}}: \underline{\underline{\hat{G}}}^{T}\right) d \bar{V}
$$

is the rate of change of total viscous dissipation,

$$
\varphi_{p r}=\int[\underline{n} \cdot(\hat{P} \underline{\underline{I}}) \cdot \underline{\hat{v}}] d \bar{\Gamma}
$$

is the energy associated with pressure perturbation,

$$
\varphi_{v i s}=\int \nabla \cdot\left[\left(\beta+\frac{1-\beta}{Y_{b}}\right)\left(\underline{\underline{\hat{G}}}+\underline{\underline{\hat{G}}}^{\mathrm{T}}\right)\right] \cdot \underline{\hat{v}} d \bar{V}
$$

is the viscous dissipation energy term,

$$
\varphi_{e l}=-\int \nabla \cdot\left[\frac{W i}{Y_{b}} \frac{\partial \underline{\underline{\underline{\Sigma}}}}{\partial t}\right] \cdot \underline{\hat{v}} d \bar{V}
$$

is the growth rate of purely elastic stress,

$$
\varphi_{\text {relax }}=\int \nabla \cdot\left[\frac{\varepsilon W i}{1-\beta} \operatorname{tr} \underline{\underline{\underline{\hat{\tau}}}} p \stackrel{\underline{\tau}}{=} p b\right] \cdot \underline{\hat{v}} d \bar{V}
$$

is the energy associated with the changes in effective relaxation time,

$$
\varphi_{p v 1}=\int \nabla \cdot\left[\frac{W i}{Y_{b}} \underline{\hat{v}} \cdot \nabla \underline{\underline{\tau}}_{p b}\right] \cdot \underline{\hat{v}} d \bar{V}
$$

represents the coupling of the velocity perturbation with the base state stress gradient,

$$
\varphi_{p v 2}=-\int \nabla \cdot\left[\frac{W i}{Y_{b}}\left(\underline{\underline{\tau}}_{p b} \cdot \underline{\underline{\hat{G}}}+\underline{\underline{\hat{G}}}^{T} \cdot \underline{\underline{\tau}}_{p b}\right)\right] \cdot \underline{\hat{v}} d \bar{V}
$$



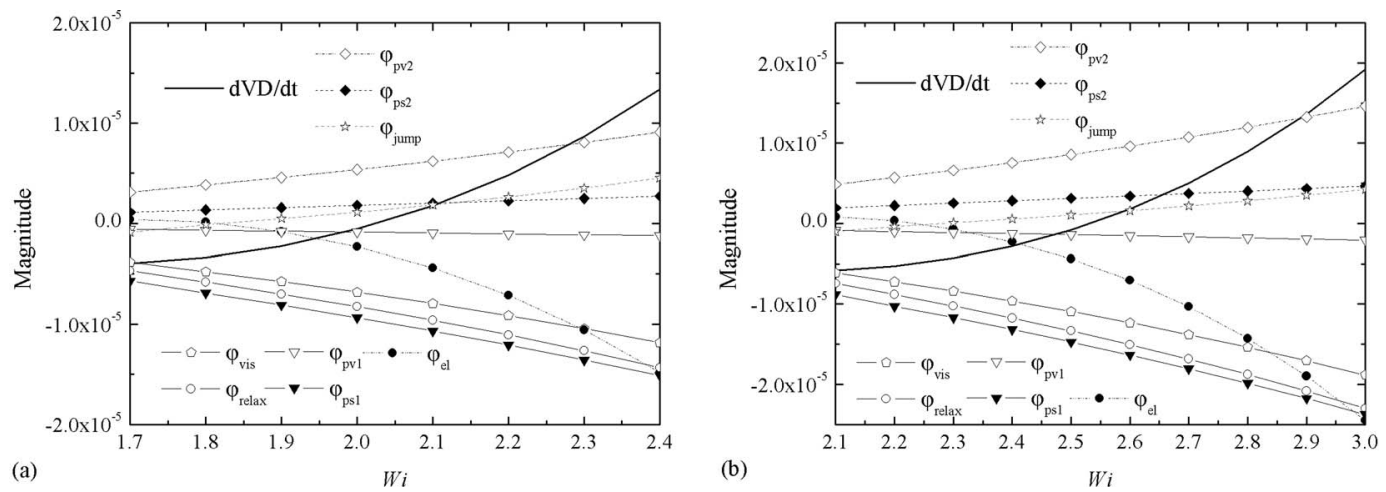

FIG. 16. Terms of the perturbation energy equation for the stick-slip flow of a PTT fluid with $\varepsilon=0.05, \beta=0$ : (a) cylindrical die and (b) planar die. Mesh M4 is used.

represents the coupling of the velocity gradient perturbation with the base state stresses,

$$
\varphi_{p s 1}=\int \nabla \cdot\left[\frac{W i}{Y_{b}} \underline{v}_{b} \cdot \nabla \underline{\underline{\hat{t}}}_{p}\right] \cdot \underline{\hat{v}} d \bar{V}
$$

represents the coupling of the stress gradient perturbation with the base state velocity,

$$
\varphi_{p s 2}=-\int \nabla \cdot\left[\frac{W i}{Y_{b}}\left(\stackrel{\underline{\hat{\tau}}}{\underline{\underline{n}}}_{p} \cdot \underline{\underline{G}}_{b}+\underline{\underline{G}}_{b}^{T} \cdot \underline{\underline{\hat{\tau}}}_{p}\right)\right] \cdot \underline{\hat{v}} d \bar{V}
$$

represents the coupling of the stress perturbation with the base state velocity gradient, and finally

$$
\varphi_{\text {jump }}=\int \underline{n} \cdot\left[W i \frac{1-\beta}{Y_{b}} \frac{\partial \underline{\underline{\underline{\hat{G}}}}}{\partial t}\right] \cdot \underline{\hat{v}} d \bar{\Gamma}
$$

represents the jump in physical properties across the interface outside the die.

Ganpule and Khomami ${ }^{53}$ have shown that the correct mode of instability can be tracked by using $d V D / d t$ as the quantity which indicates the stability or instability of the flow. Therefore, when the solvent viscosity is small or even zero as in our study, we can use the energy balance given by Eq. (13) to determine the critical couplings that cause the onset of the viscoelastic instability by examining the magnitude of each one of the terms on the right hand side near the critical $W i$ number. The driving force for the instability will be determined by finding which term(s) grow when $d V D / d t$ grows and especially which terms grow most rapidly with $W i$.

The analysis was performed for $\varepsilon=0.05$ and $\beta=0$ and for Weissenberg numbers around the critical condition. The various terms of the energy equation for both the planar and the cylindrical geometry are presented in Fig. 16, without normalization of the eigenvectors. We find that the only positive terms are $\varphi_{p v 2}, \varphi_{p s 2}$, and $\varphi_{j u m p}$, while the rest of the terms are negative for all values of $W i$. As $W i$ increases, passing the critical value, 2.03 for the cylindrical and 2.52 for the planar die (mesh M4 was used), the terms $\varphi_{p v 2}$ and $\varphi_{j u m p}$ increase significantly and seem to have a strong destabilizing effect on the flow.

On the other hand, $\varphi_{p s 2}$ appears to be smaller and increases less rapidly with $W i$ having a mild destabilizing effect as compared to the other two terms. $\varphi_{p v 2}$ represents the rate of energy production due to the coupling of velocity gradient perturbations and base state stresses, while $\varphi_{\text {jump }}$ represents the jump in physical properties across the slip interface. Considering that in stick-slip flow the streamlines of the base flow near the singularity are curved and the base and disturbance axial normal stress and the disturbance velocity gradient vary the most and in particular near the slip surface, it should not be surprising that these three terms are the ones driving the instability in this flow. Ganpule and Khomami ${ }^{53}$ found that for purely elastic instabilities in multi-layer channel flow, 
the main instability-driving terms are $\varphi_{p v 2}$ and $\varphi_{j u m p}$. In single-layer flows with curved streamlines Joo and Shaqfeh ${ }^{50,51}$ in Dean and Taylor-Couette flow and Smith et al. ${ }^{54}$ in flow around a single or an array of cylinders have reported that the observed instabilities are driven by the coupling represented by the term $\varphi_{p s 2}$, or $\varphi_{p v 1}$. Additionally, Grillet et al. ${ }^{28}$ have found that instability in planar Poiseuille or Couette flow of a PTT fluid is related to the $\varphi_{p v 2}$ term. Thus, the mechanism for the instability in stick-slip flow seems to be closer to the one reported in Ref. 53, even though in the present flow the interface is assumed to be flat corresponding to a free surface with very large surface tension. We will examine in more detail the mechanism driving the instability near the die exit, when we study this instability for the complete extrudate swell problem.

\section{Non-axisymmetric perturbations $(k \neq 0)$}

Next we examine the stability of the stick-slip flow subject to three-dimensional perturbations. To this end, we allow for harmonic disturbances in the third direction for both geometries and solve the eigenvalue problem for different wavenumbers of these perturbations. For the planar geometry no additional conditions are needed. For the cylindrical geometry, we need to pay special attention at the boundary conditions that will be imposed at the axis of symmetry of the base state now that we allow for three-dimensional perturbations $(k \neq 0)$. To this end, we follow a similar approach with Preziosi et al. ${ }^{56}$ and Miller and Rallison, ${ }^{57}$ and impose

$$
\begin{aligned}
& \hat{v}_{r}+i \hat{v}_{\theta}=0, \hat{v}_{z}=0, \hat{P}=0 \text { for } k=1, \\
& \hat{v}_{r}=0, \hat{v}_{\theta}=0, \hat{v}_{z}=0 \text { for } k>1 .
\end{aligned}
$$

Fig. 17 shows the real part of the most dangerous eigenvalue as a function of the wavenumber, $k$ for both geometries. The dispersion curves are shown for $W i$ numbers close to the critical values found for two-dimensional perturbations. From this figure we deduce that for both cylindrical and planar dies, the most unstable modes are in the range of $k=19-21$, but the pick in the growth rate is very shallow indicating a weak wave number selection. In any case, the three-dimensional instabilities are expected to appear first, something that has been observed in extrusion experiments. ${ }^{12,14,18}$ This result is a clear indication that the natural extension of this work which is to study the full extrusion problem, i.e., taking into account a deformable interface, should account for three-dimensional effects as well.

\section{Stability of the reverse flow (slip-stick flow)}

One of the three prevailing mechanisms for the sharkskin instability involves de-adhesion and slip or alternating de-adhesion and adhesion of the polymer while it is still inside the die. The preceding linear stability analysis shows that this instability may be initiated without any de-adhesion
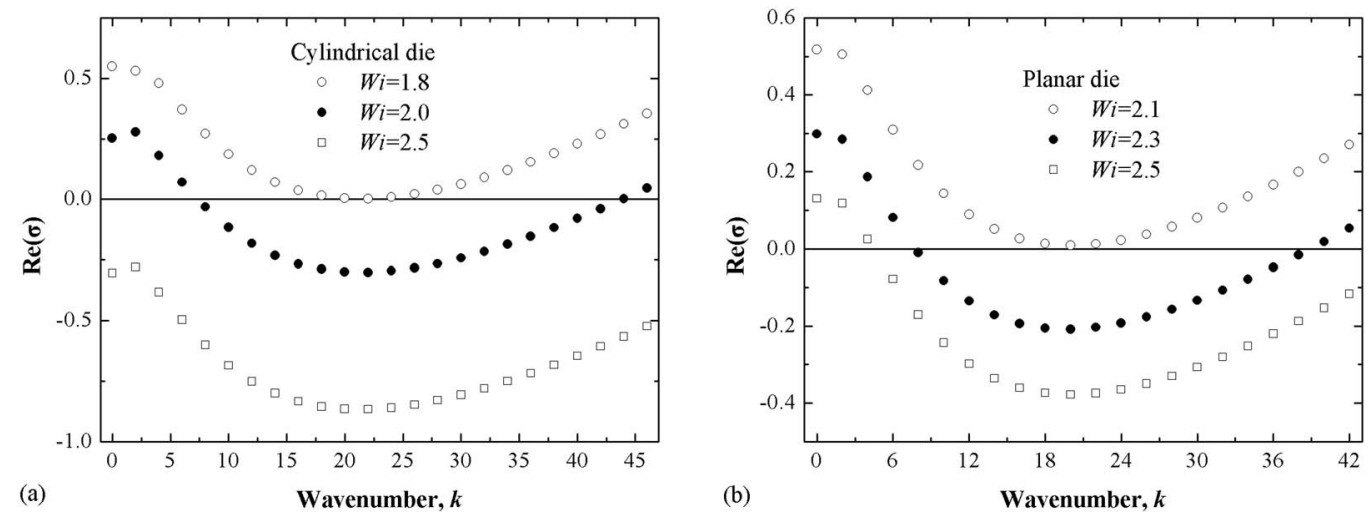

FIG. 17. Dispersion curves for non-axisymmetric perturbations for various $W i$ : (a) cylindrical die and (b) planar die. 

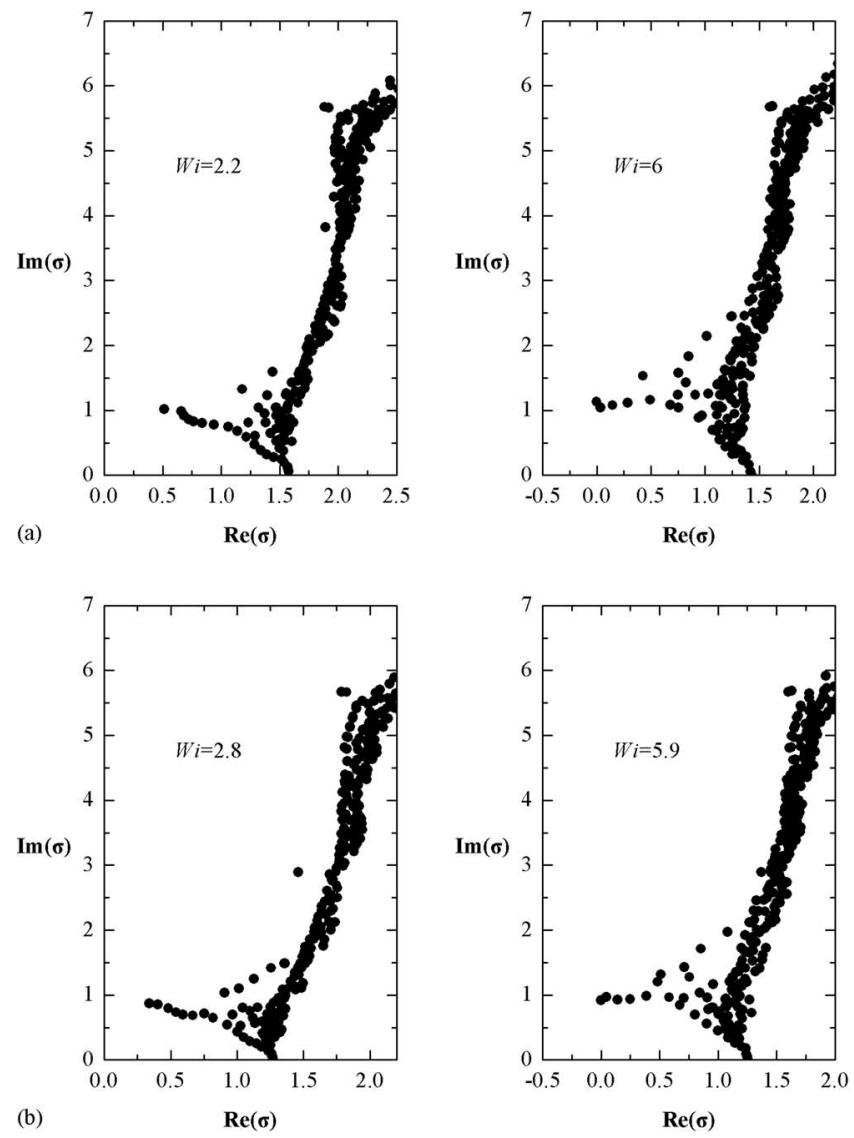

FIG. 18. Eigenspectrum for the slip-stick problem and for $\varepsilon=0.05, \beta=0, l_{1}=10, l_{2}=25$ : (a) cylindrical die and (b) planar die.

and slip inside the die. Instead, the transition from the no slip condition inside the die to the shear free condition outside it is sufficient to initiate this instability. On the other hand, the simplified flow geometry we have used allows us to examine if instability can be induced during re-adhesion of the polymer by examining the stability of the reverse flow: the slip-stick flow. In this case, the fluid will move in plug flow from the left until it enters the die and in the far field the usual outflow boundary conditions will be used to impose fully developed flow.

After calculating the steady flow and stress fields, we perform the linear stability analysis as described before. Fig. 18 shows the part of the spectrum that is closest to the imaginary axis. In both geometries, we observe that the flow remains stable for Weissenberg numbers much larger than those for which the stick-slip flow had turned unstable. In fact the slip-stick flow turns unstable for values of $W i \approx 5.9-6.0$, i.e., close to those that the 1D flow of the ePTT fluid between parallel plates or inside a tube turns unstable. ${ }^{28}$ The corresponding critical eigenvector for a planar die is shown in Fig. 19. This picture is very similar to the one for the cylindrical geometry and the latter will not be included herein. We clearly see that the critical eigenvector becomes visible well inside the die. Opposite to the stick-slip flow, here the material, as it enters the die, undergoes compression close to the wall and extension at the plane of symmetry. So the singularity in the velocity and stress components is somewhat suppressed by the flow that follows inside the die, instead of being reinforced by the locally strong extensional flow that follows the exit from the die in the stick-slip arrangement. This constitutes another indication that it is the coupling of the singularity at the die lip and the strong extensional flow near the free surface that generates the sharkskin instability. 


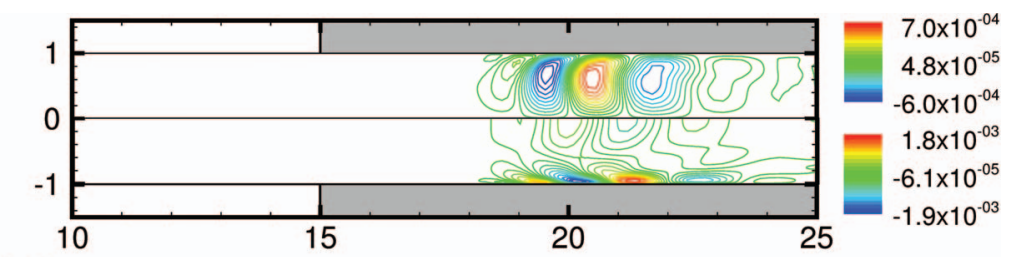

(a)

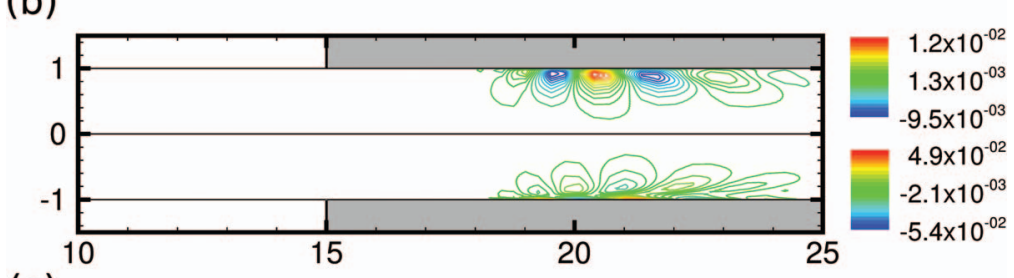

(c)

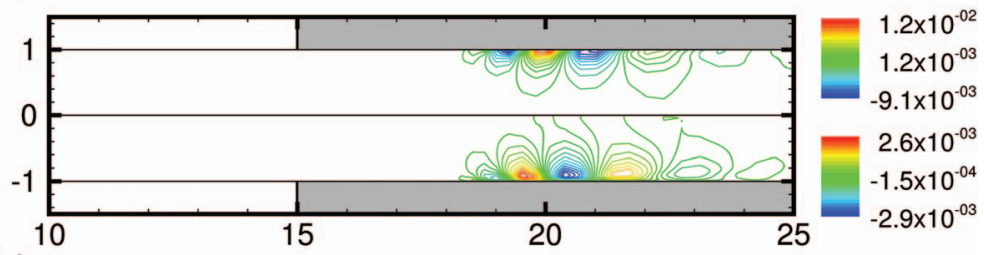

(b)

FIG. 19. Spatial form of the eigenvector for the slip-stick problem and for a planar die. Isolines of (a) $v_{r}^{\prime}, v_{z}^{\prime}$, (b) $P^{\prime}, \tau_{p r r}^{\prime}$, and (c) $\tau_{p r z}^{\prime}, \tau_{p z z}^{\prime}$ on the upper and lower half, respectively, for $W i=5.9, \varepsilon=0.05, \beta=0, l_{1}=15, l_{2}=10$ (for clarity we show the region $10 \leq z \leq 25$ ). The corresponding eigenvalue is $\sigma=-0.0003+0.9216 i$. Mesh M4 is used.

\section{CONCLUSIONS}

The purpose of this work was to study the stability of the viscoelastic stick-slip flow for a cylindrical as well as a planar geometry. In order to achieve that we performed a linear stability analysis around the steady state solution of the stick-slip flow for a viscoelastic fluid using the affine exponential PTT model. For the numerical calculations we have used the mixed finite element method combined with the EVSS-G method for the calculation of the elastic stresses together with the SUPG method for the weighting of the constitutive equation. For the calculation of the eigenvalues the Arnoldi method was employed.

The linear stability analysis around the steady state stick-slip flow of a viscoelastic fluid showed that the flow becomes unstable as the Weissenberg number increases more than a critical value, $W i_{c}$. At this critical value we have a Hopf bifurcation to a time periodic solution, the frequency of which increases with the extensional parameter, $\varepsilon$, of the PTT model and as the solvent viscosity ratio decreases. Except for the temporal periodicity the perturbed flow field also exhibits a spatial periodicity which in the corresponding extrudate swell problem could result in a wavy surface such as the one observed in experiments. Moreover we found that this critical value of $W i_{c}$, where the instability transition occurs, increases rapidly as the extensional parameter, $\varepsilon$, tends to zero approaching the stability of the UCM or the Oldroyd-B fluid models in similar flows. The same behavior of $W i_{c}$ is also found as the solvent viscosity ratio increases. Moreover, an energy analysis was performed for the most critical eigenvector in order to investigate the mechanism of the instability. It was shown that the mechanism of instability is due to the coupling of velocity gradient perturbations and base state stresses, and the jump in physical properties across the slip interface. The parametric analysis also reveals that shear and elongational thinning as well as the presence of solvent have a significant effect on the stability characteristics of the flow. When the base state is subjected to 3D disturbances, eigenmodes with wavenumber around 20 were found to be most unstable, although a range of disturbances with similar wavenumbers approach criticality at the same time. When the stability of the reverse flow is examined, the critical Weissenberg number increases, approaching the value for Poiseuille flow. 
In the literature it has been suggested that the mechanism for the instabilities should include either a non-monotonic slip law or a non-monotonic constitutive law. ${ }^{2-4,6,16,17}$ On the contrary, this work proposes that such a non-monotonic slip law or a non-monotonic constitutive law is not essential for the appearance of extrusion instabilities. Instead we show that extrusion instabilities could be due to purely elastic instabilities which can be triggered by the coupling of strong stress gradients at the die lip and the intense extensional flow, primarily at the surface of the extrudate that ensues. Our findings support at least qualitatively the mechanism for the sharkskin instability initially proposed by Cogswell. ${ }^{15}$ The present analysis examines the stick-slip flow in which the fluid volume is confined. It suggests that it is necessary to extend it allowing for the extrudate swell to take place and to examine the nonlinear and three dimensional dynamics of the flow.

\section{ACKNOWLEDGMENTS}

The authors would like to acknowledge the financial support by the General Secretariat of Research and Technology of Greece under the Action "Supporting Postdoctoral Researchers" (EIFVM, Grant No. PE8(906)) to G.K., and under the "Excellence Program" (FilCoMicrA, Grant No. 1918) to J.T., co-funded by the European Social Fund and National Resources.

\section{APPENDIX: DERIVATION OF ENERGY ANALYSIS EQUATIONS}

The disturbance energy equation is obtained by taking the inner product of the linearized perturbation of the momentum equation with the perturbation velocity and integrating the resulting equation over the volume of the flow field and one periodic cycle in time (i.e., $0<t<2 \pi / \sigma_{I}$ ).

$$
\int\left[\nabla \hat{P}-\beta \nabla \cdot\left(\underline{\underline{\hat{G}}}+\underline{\underline{\hat{G}}}^{T}\right)-\nabla \cdot \underline{\underline{\hat{\tau}}}_{p}\right] \cdot \underline{\hat{v}} d \bar{V}=0,
$$

where this notation implies the volume and time integrals of a quantity A, i.e. $\int \mathrm{A} d \bar{V}=$ $\int_{0}^{2 \pi / \sigma_{I}} \int_{V} \mathrm{~A} d V d t$. Note that for the evaluation of all terms shown in Eq. (A1) and in the equations below we take just the real part of the perturbations, $\underline{\hat{v}}, \hat{P}, \underline{\underline{\hat{G}}}$, and $\underline{\underline{\hat{\Sigma}}}$.

Because of the creeping flow assumption, the derivative of the kinetic energy of the disturbance flow does not arise in Eq. (A1), as in the standard stability analysis for Newtonian fluids. Instead, the time derivative should arise through the constitutive law. To this end, the disturbance of the polymeric part of the stress tensor, $\underline{\hat{\tau}}_{p}$, is obtained by rewriting the linearized constitutive equation as

$$
\begin{aligned}
& \underline{\hat{\tau}}_{p}=-\frac{W i}{Y_{b}}\left[\frac{\partial \underline{\underline{\hat{\tau}}}}{\partial t}+\underline{v}_{b} \cdot \nabla \frac{\hat{\underline{\tau}}}{=}+\underline{\underline{\hat{v}}} \cdot \nabla \underline{\underline{\tau}}_{p b}-\underline{\underline{\hat{\tau}}}_{p} \cdot \underline{\underline{G}}_{b}-\underline{\underline{\tau}}_{p b} \cdot \underline{\underline{\hat{G}}}-\underline{\underline{\hat{G}}}^{T} \cdot \underline{\underline{\tau}}_{p b}-\underline{\underline{G}}_{b}^{T} \cdot \underline{\hat{\tau}}_{p}\right] \\
& -W i \frac{\varepsilon}{1-\beta} \operatorname{tr} \underline{\hat{\tau}}_{p} \underline{\tau}_{p b}+\frac{1-\beta}{Y_{b}}\left(\underline{\underline{\hat{G}}}+\underline{\underline{\hat{G}}}^{T}\right)
\end{aligned}
$$

and substituting this equation into Eq. (A1) gives

$$
\frac{d E_{p}}{d t}=-\varphi_{p r}+\varphi_{v i s}-\varphi_{\text {relax }}-\varphi_{p v 1}-\varphi_{p v 2}-\varphi_{p s 1}-\varphi_{p s 2}
$$

In Eq. (A3)

$$
\frac{d E_{p}}{d t}=\int \nabla \cdot\left[\frac{W i}{Y_{b}} \frac{\partial \underline{\underline{\hat{\tau}}}}{\partial t}\right] \cdot \underline{\hat{v}} d \bar{V}
$$

is the rate of change of disturbance energy created or dissipated by the polymeric stresses,

$$
\varphi_{p r}=\int[\underline{n} \cdot(\hat{P} \underline{\underline{I}}) \cdot \underline{\hat{v}}] d \bar{\Gamma}
$$


is the energy associated with the perturbation pressure and the indicated integral implies $\int \mathrm{A} d \bar{\Gamma}=\int_{0}^{2 \pi / \sigma_{I}} \int_{\Gamma} A d \Gamma d t$

$$
\varphi_{v i s}=\int \nabla \cdot\left[\left(\beta+\frac{1-\beta}{Y_{b}}\right)\left(\underline{\underline{\hat{G}}}+\underline{\underline{\hat{G}}}^{\mathrm{T}}\right)\right] \cdot \underline{\hat{v}} d \bar{V}
$$

is the rate of viscous energy dissipation found also in the Reynolds-Orr energy equation. ${ }^{55}$ This term is always negative and, thus, it has a stabilizing effect on the flow. The following terms represent the transfer of energy from the base flow to the perturbations:

$$
\begin{gathered}
\varphi_{p v 1}=\int \nabla \cdot\left[\frac{W i}{Y_{b}} \underline{\hat{v}} \cdot \nabla_{\underline{\underline{\tau}} p b}\right] \cdot \underline{\hat{v}} d \bar{V}, \\
\varphi_{p v 2}=-\int \nabla \cdot\left[\frac{W i}{Y_{b}}\left(\underline{\underline{\tau}}_{p b} \cdot \underline{\underline{\hat{G}}}+\underline{\underline{\hat{G}}}^{T} \cdot \underline{\underline{\tau}}_{p b}\right)\right] \cdot \underline{\hat{v}} d \bar{V}, \\
\varphi_{p s 1}=\int \nabla \cdot\left[\frac{W i}{Y_{b}} \underline{\underline{v}}_{b} \cdot \nabla \underline{\underline{\hat{\tau}}}_{p}\right] \cdot \underline{\underline{\hat{v}}} d \bar{V}, \\
\varphi_{p s 2}=-\int \nabla \cdot\left[\frac{W i}{Y_{b}}\left(\underline{\underline{\hat{\tau}}}_{p} \cdot \underline{\underline{G}}_{b}+\underline{\underline{G}}_{b}^{T} \cdot \underline{\underline{\hat{\tau}}}_{p}\right)\right] \cdot \underline{\hat{v}} d \bar{V} .
\end{gathered}
$$

In the definitions of $\varphi_{p s i}, \varphi_{p v i}$, the subscripts $\mathrm{s}$ and $\mathrm{v}$ indicate that the corresponding term involves perturbations of the stress and velocity, respectively, of the upper convected terms in the constitutive law. An additional term arises in Eq. (A3) in comparison with the OLDROYD-B analysis ${ }^{28}$ from the exponential term in the PTT constitutive equation and has the following form:

$$
\varphi_{\text {relax }}=\int \nabla \cdot\left[\frac{\varepsilon W i}{1-\beta} \operatorname{tr} \underline{\underline{\hat{\tau}}} p=\underline{\underline{\tau}} p b\right] \cdot \underline{\hat{v}} d \bar{V} .
$$

This term arises due to the perturbation of the effective relaxation time.

The term $d E_{p} / d t$ has been used by several researchers for determining the mechanism of instability, e.g., Smith et al. ${ }^{54}$ In fact, Joo and Shaqfeh ${ }^{51}$ have shown that the term $d E_{p} / d t$ is related to the rate of change of the energy dissipated by the solvent, which is expressed via the positive definite norm $\beta \int \nabla v^{\prime}: \nabla v^{\prime} d V$. Hence, it plays the same role as the rate of change of the kinetic energy, which arises in finite Reynolds flows; see also Byars et al. ${ }^{52}$ Moreover, Ganpule and $\mathrm{Khomami}^{53}$ noted that this procedure is not useful when the solvent viscosity ratio is equal to zero because $d E_{p} / d t$ becomes zero as well and that additional terms should arise in multi-phase or free surface flows. In order to overcome these difficulties they have proposed to split the polymeric part of stresses in a purely viscous part and a purely elastic one (see Eq. (7)) just like the stress-splitting in the EVSS-G scheme, which we have used for the steady viscoelastic calculations in Refs. 24 and 58. This method was also used later by Grillet et al. ${ }^{28}$ Introducing that relation into Eq. (A4a) and taking into account the boundary conditions that do not set velocities at the boundaries of the slip region while integrating by parts we get

$$
\frac{d E_{p}}{d t}=-\varphi_{e l}-\frac{d V D}{d t}+\varphi_{j u m p}
$$

where

$$
\varphi_{e l}=-\int \nabla \cdot\left[\frac{W i}{Y_{b}} \frac{\partial \underline{\underline{\underline{\Sigma}}}}{\partial t}\right] \cdot \underline{\hat{v}} d \bar{V}
$$

represents the growth of purely elastic stress,

$$
\varphi_{j u m p}=\int \underline{n} \cdot\left[W i \frac{1-\beta}{Y_{b}} \frac{\partial \underline{\underline{\underline{G}}}}{\partial t}\right] \cdot \underline{\hat{v}} d \bar{\Gamma}
$$


represents the jump in physical properties across the interface outside the die, and

$$
\frac{d V D}{d t}=\int W i \frac{1-\beta}{Y_{b}} \frac{\partial}{\partial t}\left(\underline{\underline{\hat{G}}}: \underline{\underline{G}}^{T}\right) d \bar{V}
$$

is the rate of change of total viscous dissipation, similar to that originally introduced by Joo and Shaqfeh. ${ }^{51}$ Substituting Eq. (A5) into Eq. (A3) we obtain

$$
\frac{d V D}{d t}=\varphi_{p r}-\varphi_{v i s}+\varphi_{\text {relax }}+\varphi_{p v 1}+\varphi_{p v 2}+\varphi_{p s 1}+\varphi_{p s 2}-\varphi_{e l}+\varphi_{j u m p}
$$

${ }^{1}$ C. J. S. Petrie and M. M. Denn, "Instabilities in polymer processing," AIChE J. 22, 209-236 (1976).

${ }^{2}$ M. M. Denn, "Issues in viscoelastic fluid mechanics," Annu. Rev. Fluid Mech. 22, 13-34 (1990).

${ }^{3}$ M. M. Denn, "Extrusion instabilities and wall slip," Annu. Rev. Fluid Mech. 33, 265-287 (2001).

${ }^{4}$ R. G. Larson, "Instabilities in viscoelastic flows," Rheol. Acta 31, 213-263 (1992).

${ }^{5}$ M. D. Graham, "The sharkskin instability of polymer melt flows," Chaos 9, 154-163 (1999).

${ }^{6}$ S. G. Hatzikiriakos and K. Migler, Polymer Processing Instabilities: Control and Understanding (Marcel Dekker, New York, 2005).

${ }^{7}$ R. Koopmans, J. den Doelder, and J. Molenaar, Polymer Melt Fracture (CRC Press, Boca Raton, 2011).

${ }^{8}$ A. V. Ramamurthy, "Wall slip in viscous fluids and influence of materials of construction," J. Rheol. 30, 337-357 (1986).

${ }^{9}$ D. S. Kalika and M. M. Denn, "Wall slip and extrudate distortion in linear low-density polyethylene," J. Rheol. 31, 815-834 (1987).

${ }^{10}$ J. M. Piau, N. El Kissi, and B. Tremblay, "Influence of upstream instabilities and wall slip on melt fracture and sharkskin phenomena during silicones extrusion trough orifice dies," J. Non-Newtonian Fluid Mech. 34, 145-180 (1990).

${ }^{11}$ N. El Kissi and J. M. Piau, "Stability phenomena during polymer melt extrusion," J. Rheol. 38, 1447-1463 (1994).

${ }^{12}$ N. El Kissi, J. M. Piau, and F. Toussaint, "Sharkskin and cracking of polymer melt extrudates," J. Non-Newtonian Fluid Mech. 68, 271-290 (1997).

${ }^{13}$ G. Pomar, S. J. Muller, and M. M. Denn, "Extrudate distortions in linear low-density polyethylene solutions and melt," J. Non-Newtonian Fluid Mech. 54, 143-151 (1994).

${ }^{14}$ C. Venet and B. Vergnes, "Experimental characterizations of sharkskin in polyethylenes," J. Rheol. 41, 873-892 (1997).

${ }^{15}$ F. N. Cogswell, "Stretching flow instabilities at the exits of extrusion dies," J. Non-Newtonian Fluid Mech. 2, 37-47 (1977).

${ }^{16}$ G. C. Georgiou and M. J. Crochet, "Time-dependent compressible extrudate-swell problem with slip at the wall," J. Rheol. 38, 1745-1755 (1994).

${ }^{17}$ E. Brasseur, M. M. Fyrillas, G. C. Georgiou, and M. J. Crochet, "The time-dependent extrudate-swell problem of an Oldroyd-B fluid with slip along the wall,” J. Rheol. 42, 549-566 (1998).

${ }^{18}$ K. B. Migler, Y. Son, F. Qiao, and K. Flynn, "Extensional deformation, cohesive failure and boundary conditions during sharkskin melt fracture," J. Rheol. 46, 383-400 (2002).

${ }^{19}$ T. C. B. McLeish and R. C. Ball, "A molecular approach to the spurt effect in polymer melt flow," J. Polym. Sci. 24, 1735 (1986).

${ }^{20}$ G. C. Georgiou and D. Vlassopoulos, "On the stability of the simple shear flow of a Johnson-Segalman fluid," J. Non-Newtonian Fluid Mech. 75, 77-97 (1998).

${ }^{21}$ K. E. P. Adewale and A. I. Leonov, "On modelling spurt flows of polymers," J. Non-Newtonian Fluid Mech. 49, 133-138 (1993).

${ }^{22}$ E. S. G. Shaqfeh, "Purely elastic instabilities in viscometric flows," Annu. Rev. Fluid Mech. 28, 129-185 (1996).

${ }^{23}$ W. B. Black and M. D. Graham, "Effect of wall slip on the stability of viscoelastic plane shear flow," Phys. Fluids 11, 1749-1756 (1999).

${ }^{24}$ G. Karapetsas and J. Tsamopoulos, "On the stick-slip flow from slit and cylindrical dies of a Phan-Thien and Tanner fluid model. I. Steady state," Phys. Fluids 21, 123101 (2009).

${ }^{25}$ R. Natarajan and A. Acrivos, "The instability of the steady flow past sphere and disks," J. Fluid Mech. 254, 323-344 (1993).

${ }^{26}$ R. Sureshkumar and A. N. Beris, "Linear stability analysis of viscoelastic Poiseuille flow using an Arnoldi-based orthogonalization algorithm," J. Non-Newtonian Fluid Mech. 56, 151-182 (1995).

${ }^{27}$ M. S. Carvalho and L. E. Scriven, "Three-dimensional stability analysis of free surface flows: Application to forward deformable roll coating," J. Comput. Phys. 151, 534-562 (1999).

${ }^{28}$ A. M. Grillet, A. C. B. Bogaerds, G. W. M. Peters, and F. P. T. Baaijens, "Stability analysis of constitutive equations for polymer melts in viscometric flows," J. Non-Newtonian Fluid Mech. 103, 221-250 (2002).

${ }^{29}$ M. D. Smith, R. C. Armstrong, R. A. Brown, and R. Sureshkumar, "Finite element analysis of two-dimensional viscoelastic flows to three-dimensional perturbations," J. Non-Newtonian Fluid Mech. 93, 203-244 (2000).

${ }^{30}$ G. Karapetsas, O. K. Matar, P. Valluri, and K. Sefiane, "Convective rolls and hydrothermal waves in evaporating sessile drops," Langmuir 28, 11433 (2012).

${ }^{31}$ N. Phan-Thien and R. I. Tanner, "A new constitutive equation derived from network theory," J. Non-Newtonian Fluid Mech. 2, 353-365 (1977).

${ }^{32}$ N. Phan-Thien, “A nonlinear network viscoelastic model," J. Rheol. 22, 259-283 (1978).

${ }^{33}$ R. A. Brown, M. J. Szady, P. J. Northey, and R. C. Armstrong, "On the numerical stability of mixed finite-element methods for viscoelastic flows governed by differential constitutive equations," Theor. Comput. Fluid Dyn. 5, 77-106 (1993). 
${ }^{34}$ Y. Saad, "Variations of Arnoldi's method for computing eigenelements of large unsymmetric matrices," Linear Algebr. Appl. 34, 269-295 (1980).

${ }^{35}$ R. B. Lehoucq, D. C. Sorensen, and C. Yang, ARPACK User's Guide: Solution of Large Scale Eigenvalue Problems with Implicitly Restarted Arnoldi Methods (SIAM, Philadelphia, PA, 1998).

${ }^{36}$ R. Natarajan, "An Arnoldi-based iterative scheme for nonsymmetric matrix pencils arising in finite element stability problems," J. Comput. Phys. 100, 128-142 (1992).

${ }^{37}$ O. Schenk and K. Gärtner, "Solving unsymmetric sparse systems of linear equations with PARDISO," Future Gener. Comput. Syst. 20, 475-487 (2004).

${ }^{38}$ O. Schenk and K. Gärtner, "On fast factorization pivoting methods for symmetric indefinite systems," Electron. Trans. Numer. Anal. 23, 158-179 (2006), http://etna.mcs.kent.edu/volumes/2001-2010/vol23/abstract.php?vol=23\&pages=158-179.

${ }^{39}$ V. A. Gorodtsov and A. I. Leonov, "On a linear instability of a plane parallel Couette flow of viscoelastic fluid," J. Appl. Math. Mech. 31, 310-319 (1967).

${ }^{40}$ M. Renardy and Y. Renardy, "Linear stability of plane Couette flow of an upper convected Mawell fluid,” J. Non-Newtonian Fluid Mech. 22, 23-33 (1986).

${ }^{41}$ H. J. Wilson, M. Renardy, and Y. Renardy, "Structure of the spectrum in zero Reynolds number shear flow of the UCM and Oldroyd-B liquids," J. Non-Newtonian Fluid Mech. 80, 251-268 (1999).

${ }^{42}$ M. D. Graham, "Effect of axial flow on viscoelastic Taylor-Couette instability," J. Fluid Mech. 360, 341-374 (1998).

${ }^{43}$ A. C. B. Bogaerds, personal communication (2009).

${ }^{44}$ A. S. Palmer and T. N. Phillips, "Spectra approximation of Phan-Thien Tanner liquids," Numer. Algorithms 38, 133-153 (2005).

${ }^{45}$ R. A. Keiller, "Numerical instability of time dependent flows," J. Non-Newtonian Fluid Mech. 43, 229-246 (1992).

${ }^{46}$ T. C. Papanastasiou, N. Malamataris, and K. Ellwood, "A new outflow boundary condition," Int. J. Numer. Methods Fluids 14, 587-608 (1992).

${ }^{47}$ M. R. Mackley, R. P. Rutgers, and D. G. Gilbert, "Surface instabilities during the extrusion of linear low density polyethylene," J. Non-Newtonian Fluid Mech. 76, 281-297 (1998).

${ }^{48}$ G. V. Vinogradov and A. Ya. Malkin, Rheology of Polymers (Springer, Berlin, 1980).

${ }^{49}$ G. Sornberger, J. C. Quantin, R. Fajolle, B. Vergnes, and J. F. Agassant, "Experimental study of the sharkskin defect in linear low density polyethylene," J. Non-Newtonian Fluid Mech. 23, 123-135 (1987).

${ }^{50}$ Y. L. Joo and E. S. G. Shaqfeh, "Viscoelastic Poiseuille flow through a curved channel: A new elastic instability," Phys. Fluids A 3, 1691-1694 (1991).

${ }^{51}$ Y. L. Joo and E. S. G. Shaqfeh, "A purely elastic instability in Dean and Taylor-Dean flow," Phys. Fluids A 4, 524-543 (1992).

52 J. A. Byars, A. Oztekin, R. A. Brown, and G. H. McKinley, "Spiral instabilities in the flow of highly elastic fluids between rotating parallel disks," J. Fluid Mech. 271, 173-218 (1994).

${ }^{53}$ H. K. Ganpule and B. Khomami, "An investigation of interfacial instabilities in the superposed channel flow of viscoelastic fluids," J. Non-Newtonian Fluid Mech. 81, 27-69 (1999).

${ }^{54}$ M. D. Smith, Y. L. Joo, R. C. Armstrong, and R. A. Brown, "Linear stability analysis of flow of an Oldroyd-B fluid through a linear array of cylinders," J. Non-Newtonian Fluid Mech. 109, 13-50 (2003).

${ }^{55}$ P. G. Drazin and W. H. Reid, Hydrodynamic Stability (Cambridge University Press, Cambridge, UK, 1981)

${ }^{56}$ L. Preziosi, K. Chen, and D. D. Joseph, "Lubricated pipelining: stability of the core-annular flow," J. Fluid Mech. 201, 323-356 (1989).

${ }^{57}$ J. C. Miller and J. M. Rallison, "Instability of coextruded elastic liquids at high Weissenberg number," J. Non-Newtonian Fluid Mech. 143, 88-106 (2007).

${ }^{58}$ G. Karapetsas and J. Tsamopoulos, "Steady extrusion of viscoelastic materials from an annular die," J. Non-Newtonian Fluid Mech. 154, 136-152 (2008). 